\title{
Genital Chlamydia trachomatis and Neisseria gonorrhoeae infections among women in sub-Saharan Africa: A structured review
}

\author{
Jan Henk Dubbink ${ }^{1,2,3}$, Stephan P Verweij ${ }^{2}$, Helen E Struthers ${ }^{1,4}$, Sander Ouburg ${ }^{2}$, \\ James A Mclntyre ${ }^{1,5}$, Servaas A Morre ${ }^{2,3}$ and Remco PH Peters ${ }^{1,6}$
}

\begin{abstract}
Chlamydia trachomatis and Neisseria gonorrhoeae constitute major public health problems among women, but the burden of infection in sub-Saharan Africa is poorly documented. We conducted a structured review of the prevalence and incidence of genital, oral and anal C. trachomatis and $N$. gonorrhoeae infection in women in sub-Saharan Africa. We searched Medline, EMBASE and Web of Science over a 10 -year period for studies on epidemiology of genital, oral and anal chlamydial infection and gonorrhoea in women in all countries of sub-Saharan Africa. We assessed geographic and demographic differences in prevalence and incidence of infection; weighted mean prevalence estimates were calculated with a random-effect model. A total of 102 study results were included, with data available for 24/49 of sub-Saharan countries. The weighted prevalence of chlamydial infection was lower among women in community-based studies $(3.9 \%$; $95 \% \mathrm{Cl}: 2.9-5.1 \%)$ than for women recruited at primary healthcare facilities $(6.0 \% ; 95 \% \mathrm{Cl}: 4.2-8.4 \%, \mathrm{p}<0.00 \mathrm{I})$; the same was observed for gonorrhoea $(2.2 \%$; $95 \% \mathrm{Cl}$ : I.2-4.0\% vs. $4.2 \% ; 95 \% \mathrm{Cl}: 3.2-5.6 \%, \mathrm{p}<0.00 \mathrm{I})$. Prevalence of Chlamydia among sex workers was 5.5\% (95\% Cl: $4.2-7.3 \%$ ) and gonorrhoea 7.6\% (95\% Cl: 5.4-II\%). Seven studies reported on incidence which varied between $0.75-28$ and 2.8-17 per 100 person-years-at-risk for chlamydial infection and gonorrhoea, respectively. Only two studies reported on anal infections and one on oral infection. This overview underscores the considerable incidence and prevalence of genital $C$. trachomatis and $N$. gonorrhoeae in women in different settings in sub-Saharan Africa. Better control strategies are warranted to reduce the burden of infection and to prevent long-term complications of these infections.
\end{abstract}

\section{Keywords}

Chlamydia, gonorrhoea, epidemiology, sub-Saharan Africa, women, low-resource setting, sexually transmitted infections

\footnotetext{
'Anova Health Institute, Johannesburg, South Africa

${ }^{2}$ Department of Medical Microbiology \& Infection Control, Laboratory of Immunogenetics, VU University Medical Center, Amsterdam, the Netherlands

${ }^{3}$ Faculty of Health, Medicine \& Life Sciences, Department of Genetics and Cell Biology, Institute for Public Health Genomics (IPHG), Research School GROW (School for Oncology \& Developmental Biology), University of Maastricht, Maastricht, the Netherlands

${ }^{4}$ Division of Infectious Diseases \& HIV Medicine, Department of Internal Medicine, University of Cape Town, Cape Town, South Africa
}

\footnotetext{
${ }^{5}$ School of Public Health and Family Medicine, University of Cape Town, Cape Town, South Africa

${ }^{6}$ Department of Medical Microbiology, University of Pretoria, Pretoria,
} South Africa

Corresponding author:

Remco PH Peters, Anova Health Institute, 12 Sherborne Road,

Parktown, Johannesburg, South Africa.

Email: rph.peters@gmail.com 


\section{Introduction}

Chlamydia trachomatis and Neisseria gonorrhoeae are the most common bacterial sexually transmitted infections (STIs) worldwide. The most recent World Health Organization (WHO) estimates for the African region are an annual incidence (per 100 women) of 2.2 and 5.0 and prevalence ( $\%$ ) of 2.6 and 2.3 for $C$. trachomatis and $N$. gonorrhoeae infections in women aged 15-49 years in 2008, respectively. ${ }^{1}$ These regional estimates are the highest in the world, and recent modelling analysis classified sub-Saharan Africa as the only region in the high STI incidence/prevalence category. ${ }^{1,2}$

Women are more likely to be infected than men for several reasons. First, transmission is more effective from men to women due to differences between female and male susceptibility of the genital tract for infection. ${ }^{3,4}$ Second, gender inequality is especially common in resource-poor settings and places women in a more vulnerable position, e.g. for negotiating condom use or experiencing coercion. ${ }^{5}$ Finally, infection in women occurs more frequently without symptoms and is therefore more often left untreated; the majority of these infections in women are asymptomatic. $^{6,7}$ Women with unrecognized infection have an increased risk for pelvic inflammatory disease with its complications of ectopic pregnancy, tubal infertility and chronic pelvic pain. ${ }^{8}$ Furthermore, in addition to epidemiological evidence, it is biologically plausible that these STIs enhance HIV acquisition and might facilitate transmission. ${ }^{9,10}$ The identification and management of these STIs and other reproductive tract infections were identified as critical components for quality sexual and reproductive health services and have been incorporated into existing services in several countries in Africa.

As microbiological diagnosis of STIs is generally unavailable in low resource settings, the WHO recommends a syndrome-based approach to manage individuals with STI-associated symptoms. The benefits and disadvantages of this syndromic approach are well documented. ${ }^{7,11}$ An important drawback is that many infections remain untreated as they are asymptomatic whereas overtreatment with resultant unnecessary use of antibiotics also occurs, thereby enhancing risk of antimicrobial resistance. ${ }^{6,12}$ Although many countries report on the prevalence of syndromes, data about distribution of bacterial STIs in sub-Saharan African countries are generally scattered. Moreover, the currently available overviews focus on a single country or specific populations at risk, e.g. female sex workers. ${ }^{13-15}$

To our knowledge, there is no recent overview of the distribution of genital $C$. trachomatis and $N$. gonorrhoeae in women in sub-Saharan Africa. This structured review aims to inform health policies and STI control strategies for women living in low-resource settings by providing insight into the distribution of genital, oral and anal C. trachomatis and $N$. gonorrhoeae infections in women over the past 10 years across subSaharan Africa.

\section{Methods}

\section{Search strategy and selection criteria}

We searched for studies that report on the epidemiology of $C$. trachomatis and $N$. gonorrhoeae in women in subSaharan Africa, using a systematic review approach and report in accordance with the PRISMA guidelines. ${ }^{16} \mathrm{We}$ used Medline (through PubMed), EMBASE and Web of Science and searched for studies from 1 January 2005 to 31 December 2014 using two different strategies. First, the MeSH-terms and keywords 'Chlamydia trachomatis', 'Chlamydia', 'Neisseria gonorrhoeae' and 'Gonorrh(o)ea' combined with 'sub-Saharan Africa' or 'Africa South of the Sahara' (PubMed) were used. For the second search we used the same words as free text combined with the name of one of the 49 countries in sub-Saharan Africa as per combined definition of the World Health Organization (WHO) and the World Bank. ${ }^{17,18}$ We did not search conference abstracts or grey literature for additional studies. No language restrictions were applied.

\section{Study selection and eligibility criteria}

The following protocol was used to retrieve studies to be included in this review. Reference lists were generated, and titles and abstracts were independently screened by two authors (RPHP and JHD) to identify relevant articles on $C$. trachomatis and $N$. gonorrhoeae in women in sub-Saharan Africa (Figure 1). All records identified for further evaluation by at least one of the two authors were checked for duplicates and included for full text screening. The retrieved full text articles were then independently reviewed by two investigators (SPV and JHD) with discordant views resolved by referral to a third investigator (RPHP).

The following inclusion criteria were used: studies reporting on incidence and/or prevalence of $C$. trachomatis and/or $N$. gonorrhoeae in a study sample of at least 100 women recruited at one of the following settings: (a) in the general community, (b) at primary healthcare $(\mathrm{PHC})$ facilities (these include: family planning (FP), antenatal care (ANC), PHC clinic, youth clinics, HIV clinic for voluntary counselling and testing (VCT)) or (c) visiting a sexually transmitted diseases (STD) clinic. Furthermore, studies of female commercial sex workers (FSWs) and women working in a bar/ bottleshop were evaluated as high-risk groups. No age 


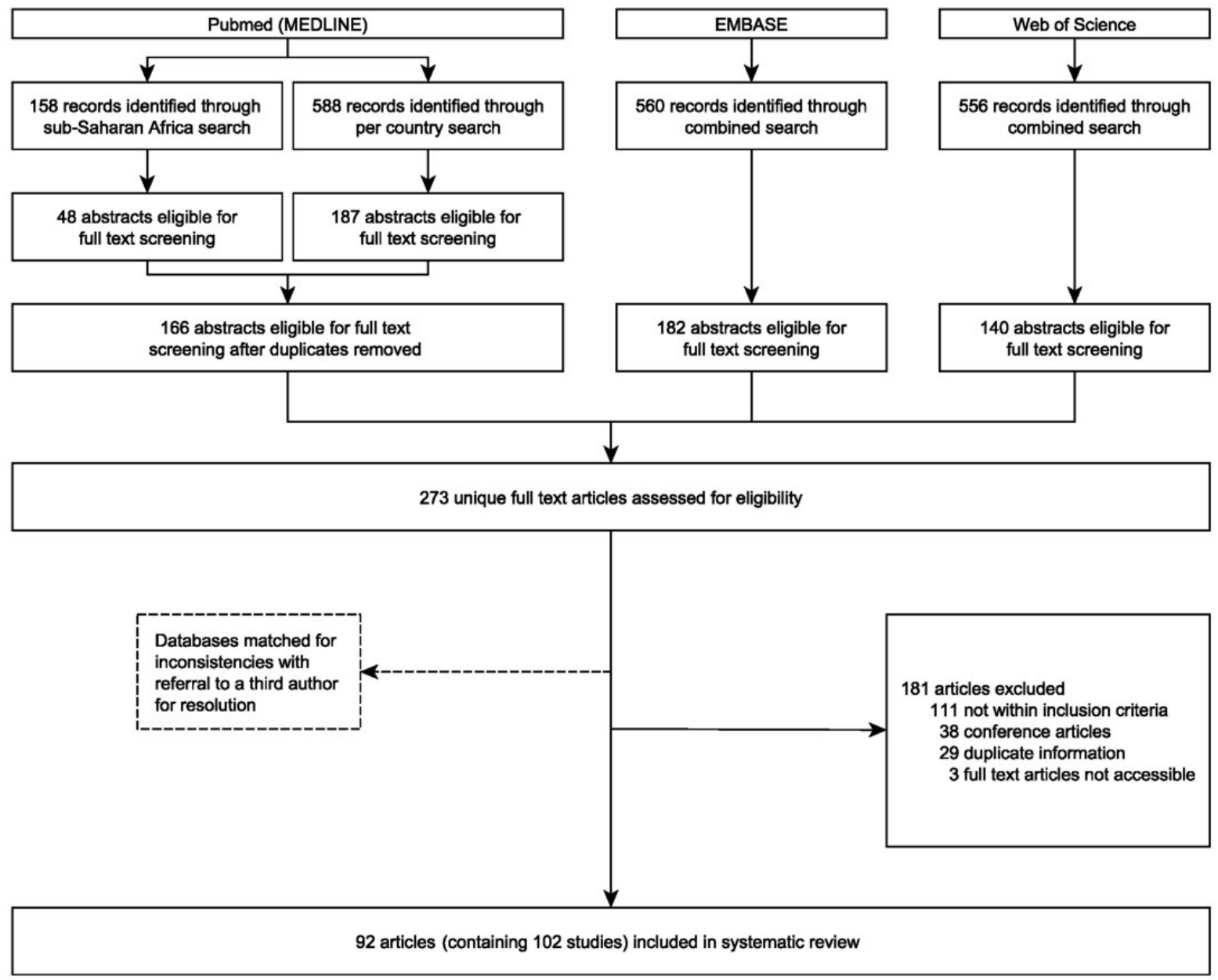

Figure I. Literature search and selection. Two searches of PubMed were conducted and a combined search was used for EMBASE and Web of Science.

restriction was applied and the review was limited to genital, anal and oral infections; Chlamydia trachomatis and Neisseria gonorrhoeae infections of other body sites (e.g. eye, joint) were excluded. Studies were only included if one of the following laboratory methods was used: nucleic acid amplification test (NAAT) for $C$. trachomatis and culture or NAAT for $N$. gonorrhoeae. Studies were excluded from further analysis based on: study design (review articles, case reports, comments on other researchers' work, studies using modelling for estimates, and case-control studies without baseline data), study objectives (general microbiology only [e.g. resistance pattern] and ocular infection), specimens used for diagnostic testing (any type other than urine, vaginal, endocervical, oropharyngeal or anorectal swab) and missing information about laboratory method or specimen. Measures of outcome were prevalence number of women with a positive test divided by total number of women with a test with interpretable result) and incidence (the proportion of all women that were negative at baseline but became infected over a defined period of time) of C. trachomatis and $N$. gonorrhoeae infection.

In the event that data from one study were reported in two or more publications, we selected the most relevant paper with regard to our objectives. For one study with no differentiation between the prevalence of Chlamydia infection and gonorrhoea and for one study with no differentiation of infection between men and women we contacted the authors for detailed information. ${ }^{19,20}$ In the case of a study involving multiple countries and/or study populations, available data were split accordingly for analysis and were eligible if each setting or risk group had a population of $\geq 100$ women. Trials were only included if baseline data were presented, as interventions could potentially bias the results.

\section{Data extraction and analysis}

Data were independently extracted by two authors (SPV and JHD) using Microsoft Access version 2013 
(Microsoft Corporation, Redmond, WA). We extracted the following variables from each article: country, year of publication, setting, population, laboratory material and method, population size (n), HIV prevalence if given, mean or median age if given, and genital, anorectal and oropharyngeal Chlamydia and/ or gonorrhoea prevalence/incidence. Both databases were compared for quality of entry with referral of discordant entries to a third author (RPHP) for resolution.

For data analysis, each country was assigned a socioeconomic status, based on the UN Human Development Index (HDI) of 2014: a composite index measuring average achievement in three basic dimensions (life expectancy, education and standard of living) of human development and ranges from very high, to high, to medium and to low. ${ }^{21}$ Furthermore, to link our data to the HIV burden in the continent, we used the estimated HIV prevalence among adults (1549 years) and in sex workers reported by UNAIDS in 2014. ${ }^{22,23}$

Data were analysed using SPSS version 20.0 (SPSS Inc, Chicago, IL) and R software package 3.3. Overview of C. trachomatis and N. gonorrhoeae prevalence per setting and population was created using maps made by ArcGIS (Esri, Redlands, CA). Per population group the weighted prevalence was calculated with corresponding 95\% confidence intervals (CIs) using the random-effect model including statistical analysis of heterogeneity. The association between the prevalence of $C$. trachomatis and $N$. gonorrhoeae infection, and between these STIs and estimated HIV prevalence, was plotted using scatter plot $\left(\mathrm{R}^{2}\right.$ values are provided in the text) and calculated using linear regression analysis.

\section{Funding source statement}

There was no relevant sponsor of the study that could have played a role in design, data collection, data analysis, data interpretation, or writing of the report. The corresponding author had full access to all the data in the study and had final responsibility for the decision to submit for publication.

\section{Results}

We identified 1862 records of which 488 (26\%) were potentially relevant. Of these, 215 were duplicates leaving the remaining 273 records for full text screening. We identified 29 publications that were considered duplication of STI data from a single research study; these were excluded from further analysis. ${ }^{11,24-51}$ After eligibility assessments, 102 study results were included in this analysis, which were published in 92 articles as eight articles had data from two countries and/or study populations and one from three (Figure 1, Table 2 and S1 Table).

\section{Characteristics of studies assessing C. trachomatis and $N$. gonorrhoeae in sub-Saharan Africa}

Prevalence data were available for 24 of the 49 countries in sub-Saharan Africa with wide variation (range: 1-18) in the number of studies that was conducted per country (Figure 2). Among these, seven studies reported on incidence within six countries (Table 3). Data on C. trachomatis were reported in 84 studies and $N$. gonorrhoeae in $101 ; 81(79 \%)$ studies reported prevalence data of both infections. Median size of study population was 448 participants (range: 100-6514). Characteristics of 88 studies reporting on prevalence in women recruited in the general community, ${ }^{20,52-69}$ at PHC settings ${ }^{6,7,19,35,70-104}$ and FSWs ${ }^{105-124}$ are described in Table 1. Furthermore, we retrieved 7 studies conducted at the STD clinic $^{72,125-130}$ and 6 studies targeting bar workers, ${ }^{131-136}$ bringing the total of studies to 102 as one study included women from both PHC setting and STD clinic. ${ }^{72}$

Overall, endocervical swabs were the most frequently used for laboratory detection of C. trachomatis (44\% of studies) and $N$. gonorrhoeae (49\%), followed by vaginal swab $(29 \%$ and $26 \%)$. Overall, we did not observe a difference in prevalence between studies using urine $(26 \%)$ versus endocervical or vaginal swabs for specimen collection for the detection of $C$. trachomatis (median $5.1 \%$ vs. $5.9 \%, p=0.29$ ). Polymerase chain reaction (PCR) was the most frequently used method for detection $(75 \%$ and $54 \%)$, followed by strand displacement amplification (SDA) for Chlamydia (14\%) and culture for gonorrhoea $(23 \%)$. For $N$. gonorrhoeae infections in women, we observed an overall difference in median prevalence between culture $(1.6 \%)$ and NAAT $(4.7 \%)$ methods for detection of infection $(p=0.001)$. We observed this difference for studies that recruited women at PHC settings $(\mathrm{p}<0.001)$, but not for studies assessing the community $(p=0.31)$ or FSWs $(p=0.59)$ as only a few $(2 / 23$ and $4 / 21)$ used culture.

\section{Prevalence of C. trachomatis and N. gonorrhoeae in the community-based studies}

Prevalence of $C$. trachomatis and $N$. gonorrhoeae in women recruited in community-based studies varied across sub-Saharan Africa (Figures 3(a) and 4(a)) and is summarized in Table 2. Highest prevalences of $C$. trachomatis and $N$. gonorrhoeae was observed in Northwestern Namibia where in one study 29/209 $(12 \%)$ and $150 / 209(72 \%)$ of women living in 28 rural villages in Kaokoland were infected, respectively. ${ }^{60}$ The weighted prevalence for $C$. trachomatis was 

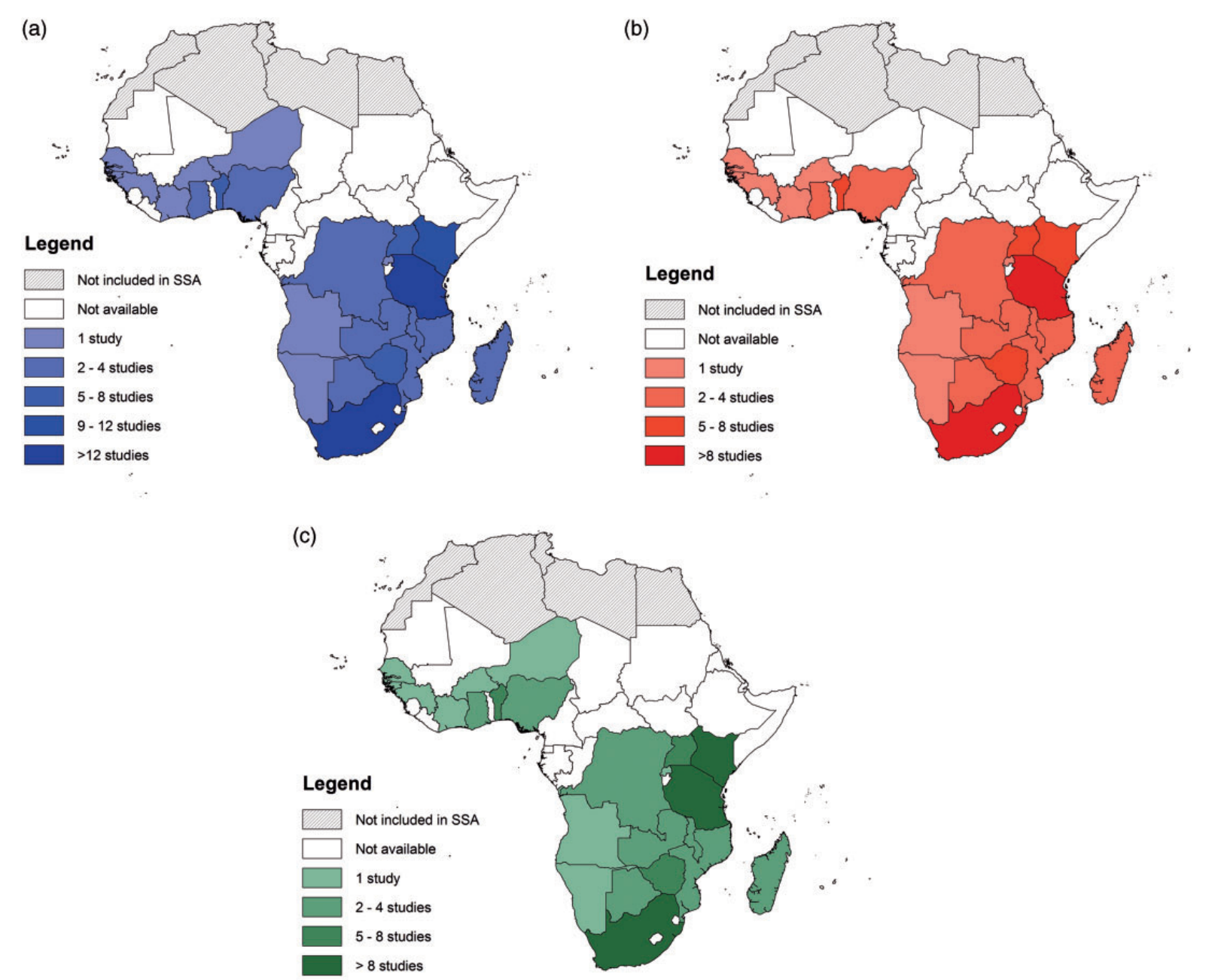

Figure 2. Number of studies reporting on the prevalence of (a) either Chlamydia trachomatis or Neisseria gonorrhoeae, (b) Chlamydia trachomatis and (c) Neisseria gonorrhoeae in women in sub-Saharan Africa as published in the period 2005-2014.

$3.9 \%\left(95 \% \mathrm{CI} ; 2.9-5.1 \% ; \mathrm{I}^{2}=95 \%\right)$ and for $N$. gonorrhoeae $2.2 \%(95 \% \mathrm{CI} ; 1.2-4.0 \%)$ in sub-Saharan Africa. When excluding the study from Hazel et al. ${ }^{60}$ because of outlier high $N$. gonorrhoeae prevalence $(72 \%)$, the weighted prevalence was $1.9 \%(95 \% \mathrm{CI}$; $1.3-2.8 \% ; \mathrm{I}^{2}=95 \%$ ) among 23850 women.

The prevalence of infection was different for the rural compared to the urban setting. For $C$. trachomatis, the prevalence was $3.0 \%(\mathrm{n}=8865)$ in the rural and $4.2 \% \quad(\mathrm{n}=13,750)$ in the urban setting $(\mathrm{p}<0.001)$ whereas for $N$. gonorrhoeae; $1.0 \%$ (8656) versus $3.6 \%$ (13989) prevalence was observed, respectively $(\mathrm{p}<0.001)$. In addition to studies conducted at the community level, prevalence of $25 \%(144 / 569)$ for chlamydial infection and 19\% (108/568) for gonorrhoea was reported among 569 young women (median age 19 years) from randomly selected high schools in rural coastal Kwazulu-Natal.

\section{Prevalence of C. trachomatis and N. gonorrhoeae in} women recruited at $\mathrm{PHC}$ settings

Thirty-three studies reported on prevalence of $C$. trachomatis and 42 for $N$. gonorrhoeae in women attending PHC facilities; 12/42 (29\%) used culture and $29 / 42(69 \%)$ used NAAT as the detection method (Table 2). Data of women recruited at PHC settings were based on the recruitment of women at primary healthcare clinics (14 studies); ANC clinics (12 studies); FP clinics (8 studies); youth clinics (5 studies) and HIV clinics (4 studies). South Africa had the highest number (13) of studies reporting on prevalence of chlamydial infection with a mean of $11 \%$ (median 14\%; range, 2.2-28\%) (Figures 3(b) and 4(b)). For $C$. trachomatis, we observed a weighted prevalence of $6.0 \%\left(95 \% \mathrm{CI} ; 4.2-8.4 \% ; \mathrm{I}^{2}=98 \%\right)$ and for $N$. gonorrhoeae a weighted prevalence of $1.0 \%(95 \% \mathrm{CI}$; $0.6-$ 
Table I. Specifications of studies included in this structured review.

\begin{tabular}{|c|c|c|c|c|}
\hline \multirow{2}{*}{ Characteristic } & \multirow{2}{*}{ Value } & \multicolumn{3}{|l|}{ No. of studies (\%) } \\
\hline & & Community $(n=23)$ & PHC setting $(n=43)$ & FSWs $(n=22)$ \\
\hline \multicolumn{5}{|l|}{ Study characteristics } \\
\hline \multirow[t]{5}{*}{ Population size (n) } & $\geq 2000$ & $3(13)$ & $6(14)$ & I (4.5) \\
\hline & $100 \mid-2000$ & $5(22)$ & $4(9.3)$ & $3(14)$ \\
\hline & $501-1000$ & 0 & II (26) & $8(36)$ \\
\hline & $20 I-500$ & $13(57)$ & $14(33)$ & $8(36)$ \\
\hline & $100-200$ & $2(8.7)$ & $8(19)$ & $2(9.1)$ \\
\hline \multirow[t]{3}{*}{ Study setting } & Urban & $13(57)$ & $36(84)$ & $22(100)$ \\
\hline & Rural & $8(35)$ & $4(9.3)$ & 0 \\
\hline & Both & $2(8.7)$ & $3(7.0)$ & 0 \\
\hline \multirow[t]{3}{*}{ Reporting on C. trachomatis } & Total & 22 & 33 & 19 \\
\hline & Prevalence data & 21 & 33 & 18 \\
\hline & Incidence data & 1 & 5 & 3 \\
\hline \multirow[t]{3}{*}{ Reporting on N. gonorrhoeae } & Total & 23 & 42 & 22 \\
\hline & Prevalence data & 22 & 42 & 21 \\
\hline & Incidence data & 2 & 5 & 2 \\
\hline \multicolumn{5}{|l|}{ Laboratory methods } \\
\hline \multicolumn{5}{|l|}{ C. trachomatis } \\
\hline \multirow[t]{4}{*}{ Collection method } & Endocervical swab & $4(17)$ & 14 (42) & II (58) \\
\hline & Vaginal swab ${ }^{a}$ & $8(35)$ & $9(27)$ & $5(26)$ \\
\hline & Urine & $10(43)$ & $9(27)$ & $3(16)$ \\
\hline & Urine and endocervical & I (4.3) & I (3.0) & 0 \\
\hline \multirow[t]{5}{*}{ Microbiological test } & NAAT & $22(100)$ & $33(100)$ & $19(100)$ \\
\hline & PCR & $14(64)$ & $26(79)$ & $14(74)$ \\
\hline & SDA & $7(32)$ & $2(6.1)$ & $2(11)$ \\
\hline & LCR & I (4.5) & $4(12)$ & $2(1 \mathrm{I})$ \\
\hline & TMA & 0 & I (3.0) & I (5.3) \\
\hline \multicolumn{5}{|l|}{ N. gonorrhoeae } \\
\hline \multirow[t]{4}{*}{ Collection method } & Endocervical swab & $4(17)$ & $20(48)$ & $18(82)$ \\
\hline & Vaginal swab ${ }^{\mathrm{a}}$ & $8(35)$ & $12(29)$ & I (4.5) \\
\hline & Urine & II (48) & $9(2 I)$ & $2(9.1)$ \\
\hline & Urine and endocervical & 0 & I (2.4) & I (4.5) \\
\hline \multirow[t]{8}{*}{ Microbiological test } & NAAT & $21(91)$ & $29(74)$ & $18(82)$ \\
\hline & PCR & $13(57)$ & $22(52)$ & 13 (59) \\
\hline & SDA & $7(30)$ & $2(4.8)$ & $2(9.1)$ \\
\hline & LCR & 0 & $4(9.5)$ & $2(9.1)$ \\
\hline & TMA & I (4.3) & I (2.4) & 0 \\
\hline & Culture & $2(8.7)$ & $12(29)$ & $4(18)$ \\
\hline & Culture and PCR & 0 & I (2.4) & 0 \\
\hline & Culture and TMA & 0 & 0 & I (4.5) \\
\hline
\end{tabular}

Note: Data of bar workers $(n=6)$ and women visiting STD clinic $(n=7)$ are not given in this table, as is one study which included women from both PHC setting and STD clinic.

PHC: primary healthcare setting; FSWs: female sex workers; PCR: polymerase chain reaction; SDA: strand displacement amplification; LCR: ligase chain reaction; TMA: transcription-mediated amplification.

${ }^{a}$ Among the 30 studies that tested women with a vaginal swab, 18 (60\%) used healthcare-worker collection and in 12 (40\%) swabs were self-collected.

$\left.1.8 \% ; \mathrm{I}^{2}=76 \%\right)$ detected by culture and $4.2 \%(95 \%$ CI; $3.2-5.6 \% ; \mathrm{I}^{2}=94 \%$ ) by NAAT.

\section{Prevalence of $C$. trachomatis and N. gonorrhoeae among FSWs and Bar workers}

Among FSWs, highest prevalence was reported in Madagascar (14\%) and Guinea (14\%) for C. trachomatis infection, and in South Africa (23\%),
Madagascar $(22 \%)$ and Uganda (13\%) for infection with $N$. gonorrhoeae (Figures 3(c) and 4(d)). Lowest prevalence was observed in Ghana (2.4\%) for chlamydial infection and in Zimbabwe $(1.9 \%)$ for gonorrhoea. We observed a weighted prevalence of $5.5 \%(95 \% \mathrm{CI}$; $4.2-7.3 \% ; \mathrm{I}^{2}=93 \%$ ) for chlamydial infection and $7.6 \%$ $\left(95 \% \mathrm{CI} ; 5.4-11 \%\right.$; $\left.\mathrm{I}^{2}=98 \%\right)$ for gonorrhoea. In addition, studies reporting on prevalence of STIs in bar workers were mainly conducted in Tanzania. Four 

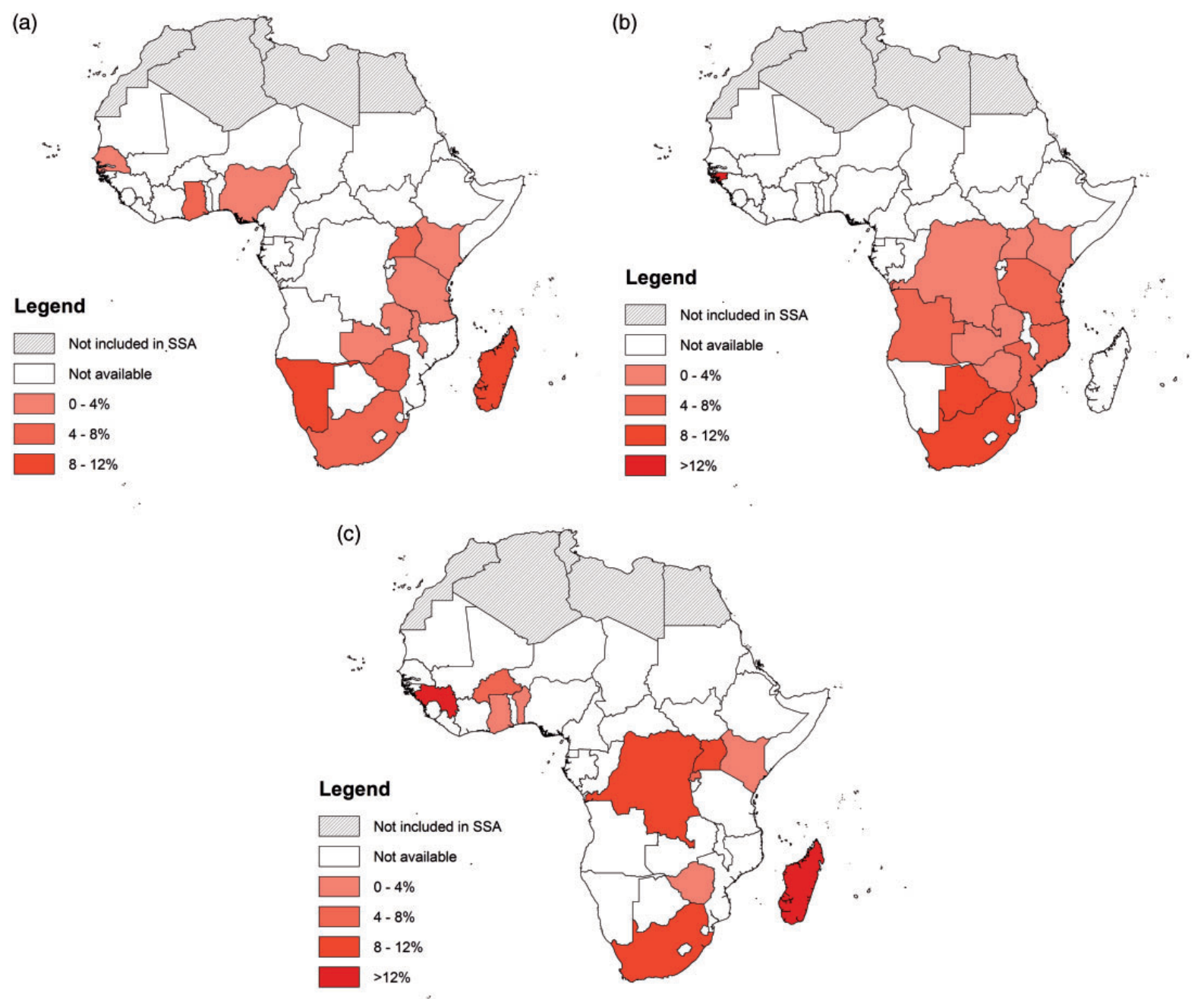

Figure 3. Prevalence of Chlamydia trachomatis reported (a) in general community settings, (b) in PHC settings and (c) among FSWs in sub-Saharan Africa. For community settings, we observed data in 21 studies in 12 countries with no studies reporting prevalence higher than I2\%; for PHC settings, we observed data in 33 studies in II countries and for FSWs, we observed data in I8 studies in II countries.

studies, all from Tanzania, reported on prevalence of chlamydial infection (range, 6.0-13\%) which gave a weighted mean of $9.0 \%(95 \% \mathrm{CI} ; 6.4-13)$. For gonorrhoea, six studies reported on prevalence of gonorrhoea (range, $0.9 \%-7.9 \%$ ) with a weighted mean of $4.8 \%$ $(95 \% \mathrm{CI} ; 2.0-11)$, including one study in Ghana with reported prevalence of $0.9 \%$ and the remainder from Tanzania.

\section{Comparison of prevalences of infection between different settings}

A higher weighted prevalence of $C$. trachomatis infection was observed at PHC settings $(6.3 \%)$ as compared to women recruited in the community $(3.9 \%$; $\mathrm{p}<0.001)$ The same difference was observed for $N$. gonorrhoeae infection with a weighted prevalence of
$4.3 \%$ at PHC facilities and $1.9 \%$ among women in the general community $(\mathrm{p}<0.001)$ The difference remained when excluding the study from Namibia as potential outlier with $72 \%$ prevalence.

In addition, weighted mean prevalence among women recruited at STD clinics was higher compared to women recruited at PHC settings for both chlamydial infection $(8.8 \%$; 95\% CI: $3.5-14 \%, \mathrm{p}<0.001)$ and gonorrhoea $(7.7 \%, 95 \% \mathrm{CI}: 4.0-14 \%, \mathrm{p}<0.001)$ based on five and seven studies, respectively. The observed prevalence among FSWs $(5.5 \%$ and $7.6 \%)$ was lower compared to the weighted prevalence among women recruited at PHC settings for chlamydial infection $(p=0.004)$ but higher for gonorrhoea $(p<0.001)$ and higher compared to women recruited in the community for both chlamydial infection and gonorrhoea $(p<0.001)$. 
(a)

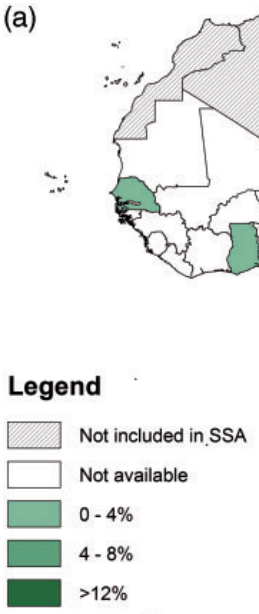

(c)

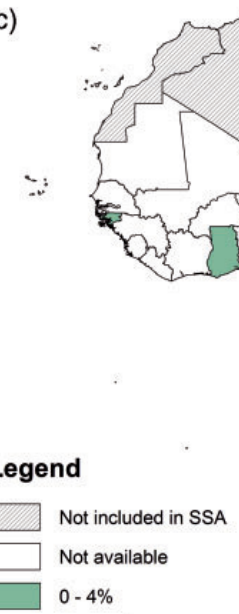

रदे

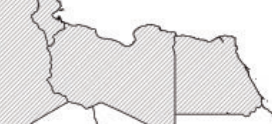

(ng

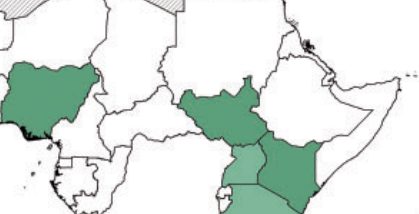

(b)

(b)

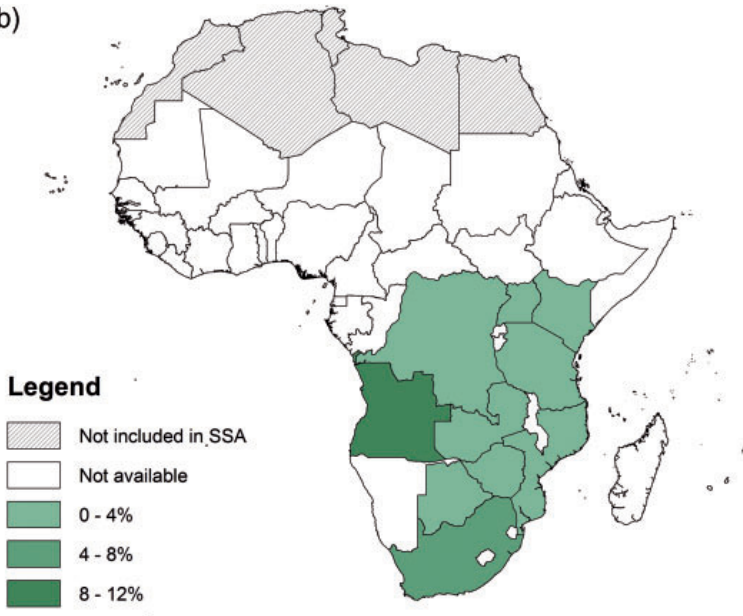

(d)

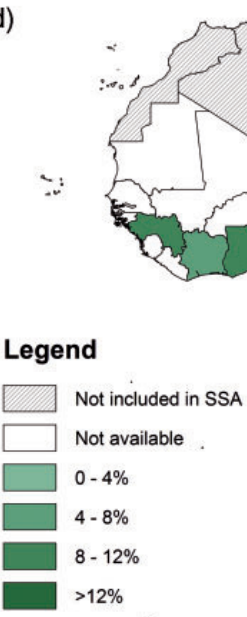

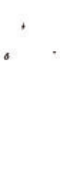

Figure 4. Prevalence of Neisseria gonorrhoeae reported (a) in general community settings, (b and c) in PHC settings and (d) among FSWs in sub-Saharan Africa. For community settings, we observed data in 22 studies in 12 countries with no studies reporting prevalence between 8 and 12\%; for PHC settings, we observed data detected by NAAT (b) in 30 studies in 10 countries and by culture (c) in 12 studies in 8 countries and for FSWs, we observed data in 21 studies in 12 countries.

\section{Incidence of C. trachomatis and N. gonorrhoeae infections}

Seven studies reported on incidence estimates of $C$. trachomatis or $N$. gonorrhoeae infections in subSaharan Africa between 2005 and 2014 (Table 3). The highest incidences of chlamydial infection (range, 0.75 28/100 person-years at risk [PYAR]) and gonorrhoea (range, 2.8-17/100 PYAR) were observed in South Africa among a family planning clinic in 2007 and among the general community in 2009 , respectively.

\section{Factors associated with prevalence of infection at population level}

We used linear regression to compare all studies $(\mathrm{n}=75)$ reporting on prevalence of both genital C. trachomatis and $N$. gonorrhoeae detected by NAAT and observed a relation $\left(p<0.0001, \mathrm{R}^{2}=0.23\right)$ between the prevalences of these two infections in sub-Saharan Africa.

Mean age of participants in studies was not associated with $C$. trachomatis $(\mathrm{p}=0.69)$ and $N$. gonorrhoeae (NAAT-based, $\mathrm{p}=0.99$ ) infection.

A higher HDI index was associated $(\mathrm{p}=0.035$, $\mathrm{R}^{2}=0.41$ ) with higher prevalence of $C$. trachomatis infection and borderline $\left(\mathrm{p}=0.078, \mathrm{R}^{2}=0.31\right)$ with higher prevalence of $N$. gonorrhoeae infection among women recruited at community level per country, but not associated with women recruited at PHC settings. After stratification for setting; we observed no association in the rural setting for HDI index with C. trachomatis $(\mathrm{p}=0.51)$ and $N$. gonorrhoeae $(\mathrm{p}=0.75)$ infection, but an association in the urban setting for both chlamydial infection $\left(\mathrm{p}=0.027 ; \mathrm{R}^{2}=0.58\right)$ and 
Table 2. Prevalences of $C$. trachomatis and $N$. gonorrhoeae in sub-Saharan Africa.

\begin{tabular}{|c|c|c|c|c|c|c|c|c|}
\hline & Author & Year & Country & Setting & $\mathrm{n}$ & Method & $\%$ & $95 \% \mathrm{Cl}$ \\
\hline \multirow[t]{45}{*}{ Community } & C. trachomatis & & & & & & & \\
\hline & Leutscher et al. $^{62}$ & 2005 & Madagascar & rural & 333 & NAAT & 9.3 & $6.6-13$ \\
\hline & Franceschi et al. ${ }^{55}$ & 2007 & Nigeria & urban & 443 & NAAT & 5.6 & $3.8-8.2$ \\
\hline & Vallely et al. ${ }^{68}$ & 2007 & Tanzania & urban & 1515 & NAAT & 5.9 & $4.8-7.2$ \\
\hline & Halpern et al. ${ }^{59}$ & 2008 & Nigeria & urban & 3619 & NAAT & 3.0 & $2.5-3.6$ \\
\hline & Mensch et al. ${ }^{63}$ & 2008 & Malawi & rural & 387 & NAAT & 1.0 & $0.4-2.7$ \\
\hline & Ghebremichael et al. ${ }^{56}$ & 2009 & Tanzania & urban & 1440 & NAAT & 1.5 & $1.0-2.3$ \\
\hline & Gueye et al. ${ }^{57}$ & 2009 & Senegal & rural & 433 & NAAT & 0.2 & $0-1.6$ \\
\hline & Doyle et al. ${ }^{54}$ & 2010 & Tanzania & rural & 6514 & NAAT & 2.4 & $2.1-2.8$ \\
\hline & Celentano et al. ${ }^{52}$ & 2010 & Zimbabwe & urban & 2943 & NAAT & 6.0 & $5.2-6.9$ \\
\hline & de Walque et al. ${ }^{20}$ & 2012 & Tanzania & both & 1205 & NAAT & 2.7 & $1.9-3.8$ \\
\hline & Paz-Soldan et al. ${ }^{66}$ & 2012 & Malawi & rural & 453 & NAAT & 5.1 & $3.4-7.6$ \\
\hline & Downs et al. ${ }^{53}$ & 2012 & Tanzania & rural & 345 & NAAT & 4.3 & $2.6-7.0$ \\
\hline & Ogilvie et al. ${ }^{64}$ & 2013 & Uganda & urban & 199 & NAAT & 14 & $9.5-19$ \\
\hline & Rutherford et al. ${ }^{67}$ & 2014 & Uganda & urban & 280 & NAAT & 2.5 & $1.2-5.1$ \\
\hline & Otieno et al. ${ }^{65}$ & 2014 & Kenya & urban & 424 & NAAT & 2.8 & $1.6-4.9$ \\
\hline & Yirenya-Tawiah et al. ${ }^{69}$ & 2014 & Ghana & rural & 191 & NAAT & 6.3 & $3.6-11$ \\
\hline & Hazel et al. ${ }^{60}$ & 2014 & Namibia & rural & 209 & NAAT & 12 & $7.8-17$ \\
\hline & Guffey et al. ${ }^{58}$ & 2014 & Malawi & urban & 1037 & NAAT & 0.5 & $0.2-1.2$ \\
\hline & Guffey et al. $^{58}$ & 2014 & South Africa & urban & 1048 & NAAT & 6.9 & $5.5-8.6$ \\
\hline & Guffey et al. ${ }^{58}$ & 2014 & Zambia & urban & 319 & NAAT & 3.1 & $1.7-5.7$ \\
\hline & Guffey et al. ${ }^{58}$ & 2014 & Zimbabwe & urban & 483 & NAAT & 3.3 & $2.0-5.3$ \\
\hline & N. gonorrhoeae & & & & & & & \\
\hline & Leutscher et al. $^{62}$ & 2005 & Madagascar & rural & 333 & culture & 5.4 & $3.4-8.4$ \\
\hline & Kapiga et al. ${ }^{61}$ & 2009 & Zambia & urban & 239 & culture & 3.4 & $1.7-6.6$ \\
\hline & Franceschi et al. $^{55}$ & 2007 & Nigeria & urban & 443 & NAAT & 2.6 & $1.5-4.6$ \\
\hline & Vallely et al. ${ }^{68}$ & 2007 & Tanzania & urban & 1515 & NAAT & 5.5 & $4.5-6.8$ \\
\hline & Halpern et al. ${ }^{59}$ & 2008 & Nigeria & urban & 3619 & NAAT & 6.1 & $5.4-6.9$ \\
\hline & Mensch et al. ${ }^{63}$ & 2008 & Malawi & rural & 387 & NAAT & 6.0 & $4.0-8.9$ \\
\hline & Ghebremichael et al. ${ }^{56}$ & 2009 & Tanzania & urban & 1440 & NAAT & 0.2 & $0.1-0.6$ \\
\hline & Gueye et al. $^{57}$ & 2009 & Senegal & rural & 433 & NAAT & 0.2 & $0-1.6$ \\
\hline & Doyle et al. ${ }^{54}$ & 2010 & Tanzania & rural & 6514 & NAAT & 0.4 & $0.3-0.6$ \\
\hline & Celentano et al. ${ }^{52}$ & 2010 & Zimbabwe & urban & 2943 & NAAT & 4.2 & $3.5-5.0$ \\
\hline & de Walque et al. ${ }^{20}$ & 2012 & Tanzania & both & 1205 & NAAT & 1.4 & $0.9-2.2$ \\
\hline & Paz-Soldan et al. ${ }^{66}$ & 2012 & Malawi & rural & 453 & NAAT & 3.3 & $2.0-5.4$ \\
\hline & Downs et al. ${ }^{53}$ & 2012 & Tanzania & rural & 345 & NAAT & 0.3 & $0-2.0$ \\
\hline & Ogilvie et al. ${ }^{64}$ & 2013 & Uganda & urban & 199 & NAAT & 1.5 & $0.5-4.6$ \\
\hline & Rutherford et al. ${ }^{67}$ & 2014 & Uganda & urban & 280 & NAAT & 1.0 & $0.3-3.2$ \\
\hline & Otieno et al. ${ }^{65}$ & 2014 & Kenya & urban & 424 & NAAT & 4.7 & $3.0-7.2$ \\
\hline & Yirenya-Tawiah et al. ${ }^{69}$ & 2014 & Ghana & rural & 191 & NAAT & 2.6 & $1.1-6.1$ \\
\hline & Hazel et al. ${ }^{60}$ & 2014 & Namibia & rural & 209 & NAAT & 72 & $65-78$ \\
\hline & Guffey et al. ${ }^{58}$ & 2014 & Malawi & urban & 1037 & NAAT & 0.9 & $0.5-1.7$ \\
\hline & Guffey et al. ${ }^{58}$ & 2014 & South Africa & urban & 1048 & NAAT & 0.9 & $0.5-1.7$ \\
\hline & Guffey et al. ${ }^{58}$ & 2014 & Zambia & urban & 319 & NAAT & 2.9 & $1.5-5.4$ \\
\hline & Guffey et al. ${ }^{58}$ & 2014 & Zimbabwe & urban & 483 & NAAT & 0.4 & $0.1-1.6$ \\
\hline \multirow[t]{10}{*}{ Primary healthcare clinic } & C. trachomatis & & & & & & & \\
\hline & Paz-Bailey et al. ${ }^{94}$ & 2005 & Botswana & Urban & 700 & NAAT & 13 & $11-16$ \\
\hline & Pettifor et al..$^{95}$ & 2005 & South Africa & Urban & 4626 & NAAT & 9.8 & $9.0-11$ \\
\hline & Pickering et al. ${ }^{96}$ & 2005 & Uganda & Rural & 206 & NAAT & 0.5 & $0.1-3.4$ \\
\hline & Rassjö et al. ${ }^{40,98}$ & 2006 & Uganda & Urban & 199 & NAAT & 4.5 & $2.4-8.4$ \\
\hline & Tann et al. ${ }^{101}$ & 2006 & Uganda & Urban & 250 & NAAT & 5.9 & $3.6-9.6$ \\
\hline & van de Wijgert et al. ${ }^{102}$ & 2006 & South Africa & Both & 450 & NAAT & II & $8.2-14$ \\
\hline & Frohlich et al. $^{75}$ & 2007 & South Africa & Rural & 274 & NAAT & 8.8 & $6.0-13$ \\
\hline & Jones et al. ${ }^{80}$ & 2007 & South Africa & Urban & 626 & NAAT & 22 & $19-25$ \\
\hline & Kleinschmidt et al. ${ }^{82}$ & 2007 & South Africa & Urban & 551 & NAAT & 14 & $11-17$ \\
\hline
\end{tabular}


Table 2. Continued

\begin{tabular}{|c|c|c|c|c|c|c|c|}
\hline Author & Year & Country & Setting & $\mathrm{n}$ & Method & $\%$ & $95 \% \mathrm{Cl}$ \\
\hline Romoren et al. $^{7}$ & 2007 & Botswana & Urban & 703 & NAAT & 8.0 & $6.2-8.8$ \\
\hline Watson-jones et al. ${ }^{104}$ & 2007 & Tanzania & Urban & 1536 & NAAT & 7.4 & $6.2-8.8$ \\
\hline Hubacher et al. ${ }^{79}$ & 2008 & South Africa & Urban & 125 & NAAT & 23 & $|7-3|$ \\
\hline Kinoshita-Moleka et al. ${ }^{81}$ & 2008 & DRC & Urban & 521 & NAAT & 1.7 & $0.9-3.3$ \\
\hline Luján et al. ${ }^{85}$ & 2008 & Mozambique & Rural & 835 & NAAT & 4.1 & $2.9-5.7$ \\
\hline Ramjee et al. $^{97}$ & 2008 & Zimbabwe & Urban & 2455 & NAAT & 2.0 & I.5-2.6 \\
\hline Ramjee et al. ${ }^{97}$ & 2008 & South Africa & Urban & 2493 & NAAT & 7.9 & $6.9-9.0$ \\
\hline Govender et al. $^{76}$ & 2009 & South Africa & Urban & 219 & NAAT & 19 & $14-24$ \\
\hline Guthrie et al. ${ }^{78}$ & 2009 & Kenia & Urban & 416 & NAAT & 0.7 & $0.2-2.2$ \\
\hline Saleh-Onoya et al. ${ }^{99}$ & 2009 & South Africa & Urban & 100 & NAAT & 13 & $7.7-21$ \\
\hline van de Wijgert et al. ${ }^{103}$ & 2009 & Zimbabwe & Urban & 2240 & NAAT & 3.0 & $2.4-3.8$ \\
\hline van de Wijgert et al. ${ }^{103}$ & 2009 & Uganda & Urban & 2199 & NAAT & 2.9 & $2.3-3.7$ \\
\hline Darj et al. ${ }^{19}$ & 2010 & Uganda & Both & 592 & NAAT & 3.5 & $2.3-5.3$ \\
\hline Mansson et al. ${ }^{86}$ & 2010 & Guinea-Bissau & Urban & 711 & NAAT & 13 & $10-15$ \\
\hline Marx et al. ${ }^{87}$ & 2010 & Kenya & Urban & 441 & NAAT & 4.3 & $2.8-6.6$ \\
\hline Mhlongo et al. ${ }^{89}$ & 2010 & South Africa & Urban & 300 & NAAT & 15 & $11-19$ \\
\hline Alcaide et al. $^{71}$ & 2012 & Zambia & Urban & 292 & NAAT & 1.0 & $0.3-3.1$ \\
\hline Chiduo et al. $^{74}$ & 2012 & Tanzania & Urban & 185 & NAAT & 1.6 & $0-4.7$ \\
\hline Lewis et al. ${ }^{84}$ & 2012 & South Africa & Urban & 558 & NAAT & 2.2 & $1.3-3.8$ \\
\hline Onoya et al. ${ }^{93}$ & 2012 & South Africa & Urban & 446 & NAAT & 28 & $24-32$ \\
\hline Guimaräes et al. $^{77}$ & 2013 & Angola & Urban & 246 & NAAT & 7.3 & $4.6-11$ \\
\hline Singa et al. ${ }^{100}$ & 2013 & Kenya & Both & 1063 & NAAT & 0.6 & $0.3-1.2$ \\
\hline Mavedzenge et al. ${ }^{35}$ & 2014 & Zimbabwe & Unclear & $|3|$ & NAAT & 4.6 & $2.1-9.8$ \\
\hline Peters et al. ${ }^{6}$ & 2014 & South Africa & Rural & 604 & NAAT & 16 & $13-19$ \\
\hline N. gonorrhoeae & & & & & & & \\
\hline Lassey et al. ${ }^{83}$ & 2005 & Ghana & Urban & 100 & Culture & 0 & NA \\
\hline Bukusi et al. $^{72}$ & 2006 & Kenya & Urban & 112 & Culture & 1.0 & $0.2-6.1$ \\
\hline Chalamilla et al. ${ }^{73}$ & 2006 & Tanzania & Urban & 101 & Culture & 3.7 & I.3-9.7 \\
\hline Msuya et al. ${ }^{90}$ & 2006 & Tanzania & Urban & 382 & Culture & 0.5 & $0.1-2.1$ \\
\hline Aboud et al. ${ }^{70}$ & 2008 & Malawi & Urban & 1222 & Culture & 3.4 & $2.5-4.6$ \\
\hline Aboud et al. ${ }^{70}$ & 2008 & Tanzania & Urban & 428 & Culture & 0.2 & $0-1.6$ \\
\hline Aboud et al. ${ }^{70}$ & 2008 & Zambia & Urban & 642 & Culture & 1.6 & $0.9-2.9$ \\
\hline Melo et al. ${ }^{88}$ & 2008 & Mozambique & Urban & 435 & Culture & 0.2 & $0-1.6$ \\
\hline Msuya et al. ${ }^{91}$ & 2009 & Tanzania & Urban & 2555 & Culture & 0.5 & $0.3-0.9$ \\
\hline Mansson et al. ${ }^{86}$ & 2010 & Guinea-Bissau & Urban & 711 & Culture & 1.3 & $0.7-2.5$ \\
\hline Nwadioha et al. ${ }^{92}$ & 2011 & Nigeria & Urban & 1000 & Culture & 0.5 & $0.2-1.2$ \\
\hline Chiduo et al. ${ }^{74}$ & 2012 & Tanzania & Urban & 185 & Culture & 1.6 & $0.5-4.9$ \\
\hline Paz-Bailey et al. ${ }^{94}$ & 2005 & Botswana & Urban & 700 & NAAT & 4.5 & $3.2-6.3$ \\
\hline Pettifor et al. ${ }^{95}$ & 2005 & South Africa & Urban & 4626 & NAAT & 3.5 & $3.0-4.1$ \\
\hline Pickering et al. $^{96}$ & 2005 & Uganda & Rural & 206 & NAAT & 7.8 & $4.8-12$ \\
\hline Rassjö et al. ${ }^{40,98}$ & 2006 & Uganda & Urban & 199 & NAAT & 9.0 & $5.7-14$ \\
\hline Tann et al. ${ }^{101}$ & 2006 & Uganda & Urban & 250 & NAAT & 4.3 & $2.2-7.7$ \\
\hline van de Wijgert et al. ${ }^{102}$ & 2006 & South Africa & Both & 450 & NAAT & 7.3 & $5.2-10$ \\
\hline Frohlich et al. ${ }^{75}$ & 2007 & South Africa & Rural & 274 & NAAT & 6.2 & 3.9-9.7 \\
\hline Jones et al. ${ }^{80}$ & 2007 & South Africa & Urban & 626 & NAAT & 8.0 & $6.1-10$ \\
\hline Kleinschmidt et al. ${ }^{82}$ & 2007 & South Africa & Urban & 551 & NAAT & 3.9 & $2.6-5.9$ \\
\hline Romoren et al. $^{7}$ & 2007 & Botswana & Urban & 703 & NAAT & 3.0 & $2.0-4.6$ \\
\hline Watson-jones et al. ${ }^{104}$ & 2007 & Tanzania & Urban & 1536 & NAAT & 2.2 & $1.6-3.1$ \\
\hline Hubacher et al. ${ }^{79}$ & 2008 & South Africa & Urban & 125 & NAAT & 6.4 & $3.2-12$ \\
\hline Kinoshita-Moleka et al. ${ }^{81}$ & 2008 & DRC & Urban & 521 & NAAT & 0.4 & $0.1-1.5$ \\
\hline Luján et al. ${ }^{85}$ & 2008 & Mozambique & Rural & 835 & NAAT & 2.5 & $1.6-3.8$ \\
\hline Ramjee et al. $^{97}$ & 2008 & Zimbabwe & Urban & 2455 & NAAT & 2.0 & I.5-2.6 \\
\hline Ramjee et al. ${ }^{97}$ & 2008 & South Africa & Urban & 2493 & NAAT & 2.1 & $1.6-2.7$ \\
\hline Guthrie et al. ${ }^{78}$ & 2009 & Kenia & Urban & 416 & NAAT & 0 & NA \\
\hline Saleh-Onoya et al. ${ }^{99}$ & 2009 & South Africa & (l) & 100 & A & 11 & $6.2-19$ \\
\hline
\end{tabular}


Table 2. Continued

\begin{tabular}{|c|c|c|c|c|c|c|c|c|}
\hline & Author & Year & Country & Setting & $\mathrm{n}$ & Method & $\%$ & $95 \% \mathrm{Cl}$ \\
\hline & van de Wijgert et al. ${ }^{103}$ & 2009 & Zimbabwe & Urban & 2240 & NAAT & 2.2 & $1.7-2.9$ \\
\hline & van de Wijgert et al. ${ }^{103}$ & 2009 & Uganda & Urban & 2199 & NAAT & 1.8 & $1.7-2.9$ \\
\hline & Darj et al..$^{19}$ & 2010 & Uganda & Both & 592 & NAAT & 4.7 & $3.3-6.7$ \\
\hline & Marx et al. ${ }^{87}$ & 2010 & Kenya & Urban & $44 I$ & NAAT & 1.6 & $0.8-3.2$ \\
\hline & Mhlongo et al. ${ }^{89}$ & 2010 & South Africa & Urban & 300 & NAAT & 14 & $11-18$ \\
\hline & Alcaide et al. ${ }^{71}$ & 2012 & Zambia & Urban & 292 & NAAT & 1.4 & $0.5-3.6$ \\
\hline & 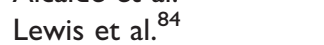 & 2012 & South Africa & Urban & 558 & NAAT & 6.4 & $4.6-8.8$ \\
\hline & Onoya et al. ${ }^{93}$ & 2012 & South Africa & Urban & 446 & NAAT & 12 & $9.4-16$ \\
\hline & Guimaräes et al. $^{77}$ & 2013 & Angola & Urban & 246 & NAAT & 8.5 & $5.6-13$ \\
\hline & Singa et al. 100 & 2013 & Kenya & Both & 1063 & NAAT & 1.6 & $0.1-2.6$ \\
\hline & Mavedzenge et al. ${ }^{35}$ & 2014 & Zimbabwe & Unclear & $|3|$ & NAAT & 9.2 & $5.3-16$ \\
\hline & Peters et al. ${ }^{6}$ & 2014 & South Africa & Rural & 604 & NAAT & 10 & $7.8-13$ \\
\hline \multirow[t]{41}{*}{ Female sex workers } & C. trachomatis & & & & & & & \\
\hline & Cohen et al. ${ }^{\prime \prime I}$ & 2005 & Kenya & Urban & 299 & NAAT & 8.0 & $5.4-12$ \\
\hline & Cowan et al. ${ }^{1 / 2}$ & 2005 & Zimbabwe & Rural & 363 & NAAT & 1.7 & $0.8-3.7$ \\
\hline & Dunkle et al. ${ }^{1 / 3}$ & 2005 & South Africa & Urban & 295 & NAAT & 8.4 & $5.7-12$ \\
\hline & Feldblum et al. ${ }^{1 / 4}$ & 2005 & Madagascar & Urban & 901 & NAAT & 15 & $12-17$ \\
\hline & Pépin et al. ${ }^{120}$ & 2005 & Ghana & Urban & 382 & NAAT & 2.4 & $1.3-4.5$ \\
\hline & Pépin et al. ${ }^{120}$ & 2005 & Benin & Urban & 444 & NAAT & 4.3 & $2.8-6.6$ \\
\hline & Alary et al. ${ }^{108}$ & 2006 & Benin & Urban & 1084 & NAAT & 4.7 & $3.6-6.1$ \\
\hline & Ahoyo et al. ${ }^{106}$ & 2007 & Benin & Urban & 723 & NAAT & 6.0 & $4.5-8.0$ \\
\hline & Vandepitte et al. ${ }^{122}$ & 2007 & DRC & Urban & 502 & NAAT & 8.4 & $6.3-11$ \\
\hline & Ahoyo et al. ${ }^{107}$ & 2009 & Benin & Urban & 620 & NAAT & 2.4 & $1.4-4.0$ \\
\hline & Harijaona et al. ${ }^{1 / 6}$ & 2009 & Madagascar & Urban & 100 & NAAT & 12 & $6.9-20$ \\
\hline & Vandepitte et al. ${ }^{121}$ & 2011 & Uganda & Urban & 1027 & NAAT & 9.0 & $7.4-11$ \\
\hline & Veldhuijzen et al. ${ }^{47}$ & 2011 & Rwanda & Urban & 396 & NAAT & 5.0 & $3.2-7.6$ \\
\hline & Vuylsteke et al. ${ }^{123}$ & 2012 & Cote d'lvoire & Urban & 1110 & NAAT & 5.2 & $4.0-6.7$ \\
\hline & Béhanzin et al. $^{109}$ & 2013 & Benin & Urban & 613 & NAAT & 3.9 & $2.6-5.8$ \\
\hline & Masese et al. ${ }^{119}$ & 2013 & Kenya & Urban & 865 & NAAT & 1.9 & $\mid .2-3.1$ \\
\hline & Aho et al. ${ }^{105}$ & 2014 & Guinea & Urban & 223 & NAAT & 14 & $9.7-19$ \\
\hline & Gomih-Alakija et al. ${ }^{115}$ & 2014 & Kenya & Urban & 349 & NAAT & 3.7 & $2.2-6.3$ \\
\hline & N. gonorrhoeae & & & & & & & \\
\hline & Mamadou et al. ${ }^{1 / 8}$ & 2006 & Niger & Urban & 529 & culture & 7.0 & $5.1-9.5$ \\
\hline & Chersich et al. ${ }^{110}$ & 2007 & Kenya & Urban & 677 & culture & 0.7 & $0.3-1.7$ \\
\hline & Harijaona et al. ${ }^{1 / 6}$ & 2009 & Madagascar & Urban & 100 & culture & 27 & $19-37$ \\
\hline & Izulla et al. ${ }^{117}$ & 2013 & Kenya & Urban & 2933 & culture & 3.1 & $2.5-3.8$ \\
\hline & Masese et al. ${ }^{199}$ & 2013 & Kenya & Urban & 865 & Both & 2.5 & $1.6-3.8$ \\
\hline & Cowan et al. ${ }^{1 / 2}$ & 2005 & Zimbabwe & Rural & 363 & NAAT & 1.9 & $0.9-4.0$ \\
\hline & Pépin et al. ${ }^{120}$ & 2005 & Ghana & Urban & 382 & NAAT & 12 & $8.9-15$ \\
\hline & Pépin et al. ${ }^{120}$ & 2005 & Benin & Urban & 444 & NAAT & 20 & $16-24$ \\
\hline & Feldblum et al. ${ }^{1 / 4}$ & 2005 & Madagascar & Urban & 901 & NAAT & 22 & $19-24$ \\
\hline & Dunkle et al. ${ }^{113}$ & 2005 & South Africa & Urban & 295 & NAAT & 23 & $19-29$ \\
\hline & Cohen et al. ${ }^{1 \mathrm{I}}$ & 2005 & Kenya & Urban & 299 & NAAT & 6.0 & $3.8-9.3$ \\
\hline & Alary et al. ${ }^{108}$ & 2006 & Benin & Urban & 1084 & NAAT & 4.6 & $3.5-6.0$ \\
\hline & Vandepitte et al. ${ }^{122}$ & 2007 & DRC & Urban & 502 & NAAT & 7.8 & $5.8-11$ \\
\hline & Ahoyo et al. ${ }^{106}$ & 2007 & Benin & Urban & 723 & NAAT & 20 & $18-24$ \\
\hline & Ahoyo et al. ${ }^{107}$ & 2009 & Benin & Urban & 620 & NAAT & 7.0 & $5.2-9.3$ \\
\hline & Vandepitte et al. ${ }^{121}$ & 2011 & Uganda & Urban & 1027 & NAAT & 13 & $11-15$ \\
\hline & Vuylsteke et al. ${ }^{123}$ & 2012 & Cote d'lvoire & Urban & 1110 & NAAT & 5.1 & $4.0-6.6$ \\
\hline & Veldhuijzen et al. ${ }^{47}$ & 2011 & Rwanda & Urban & 396 & NAAT & 12 & $8.8-15$ \\
\hline & Béhanzin et al. $^{109}$ & 2013 & Benin & Urban & 613 & NAAT & 5.9 & $4.3-8.1$ \\
\hline & Aho et al. ${ }^{105}$ & 2014 & Guinea & Urban & 223 & NAAT & 9.0 & $5.9-14$ \\
\hline & Gomih-Alakija et al. ${ }^{1 / 5}$ & 2014 & Kenya & Urban & 349 & NAAT & 2.3 & $1.2-4.5$ \\
\hline
\end{tabular}


Table 3. Incidence per 100 woman years of C. trachomatis or N. gonorrhoeae reported in sub-Saharan Africa from 2005 to 2014.

\begin{tabular}{|c|c|c|c|c|c|c|}
\hline Author & Year & Country & Group & Population & C. trachomatis & N. gonorrhoeae \\
\hline Kleinschmidt et al. ${ }^{82}$ & 2007 & South Africa & FP & 551 & 28 & 9.9 \\
\hline \multirow[t]{2}{*}{ Ramjee et al. ${ }^{97}$} & 2008 & South Africa & $\mathrm{PHC}$ & 2493 & 9.5 & 3.7 \\
\hline & 2008 & Zimbabwe & $\mathrm{PHC}$ & 2455 & 3.3 & 1.3 \\
\hline \multirow{2}{*}{ van de Wijgert et al. ${ }^{103}$} & 2009 & Uganda & $\mathrm{FP}$ & 2199 & 4.1 & 5.2 \\
\hline & 2009 & Zimbabwe & FP & 2240 & 3.7 & 3.7 \\
\hline Kapiga et al. ${ }^{61}$ & 2009 & South Africa & Community & 479 & 20 & 17 \\
\hline Veldhuijzen et al. ${ }^{124}$ & 2013 & Rwanda & FSWs & 396 & 8.1 & 12 \\
\hline Masese et al. ${ }^{119}$ & 2013 & Kenya & FSWs & 865 & 5 & NA \\
\hline Low et al. ${ }^{137}$ & 2014 & Burkina Faso & FSWs & 172 & 0.75 & 2.8 \\
\hline
\end{tabular}

FSWs: female sex workers; FP: family planning; PHC: primary healthcare clinic; NA: not available; DRC: Democratic Republic of Congo.

gonorrhoea $\left(p=0.018 ; R^{2}=0.64\right)$ with higher HDI was observed among the community-based studies.

We did not observe an association of genital C. trachomatis infection and HIV prevalence as reported in the different studies $(\mathrm{n}=44, \mathrm{p}=0.70)$, but we did observe an association of $N$. gonorrhoeae infection (detected by NAAT) with reported HIV prevalence $\left(\mathrm{n}=40, \mathrm{p}<0.0001, \mathrm{R}^{2}=0.38\right)$.

There was no relation of HIV prevalence estimates among adults (reported by UNAIDS) with prevalence of $C$. trachomatis and $N$. gonorrhoeae at community level and at PHC settings per country. Furthermore, there was no association of HIV prevalence estimates among FSWs (reported by UNAIDS) with $C$. trachomatis and $N$. gonorrhoeae infection.

\section{Oral and anal infections}

Two studies reported on anal infections among African women. Rutherford et al. ${ }^{67}$ observed of $0 \%$ prevalence for anorectal chlamydial infection and $0.45 \%$ for anorectal gonorrhoea (detected by PCR) among 280 women in Uganda. No data were reported on receptive anal intercourse (RAI) and fellatio in this study. A study from South Africa showed prevalences of 7.1\% and $0.2 \%$, and $2.5 \%$ and $0 \%$ for anorectal and oropharyngeal Chlamydia and gonorrhoea (detected by PCR), respectively, among 604 women. RAI was reported by 26 of these women $(4.6 \%)$, and fellatio by 80 women $(14 \%)$. Neither practice was associated with genital or rectal infection in that study.

\section{Discussion}

This review summarizes data from 102 studies reporting on the epidemiology of C. trachomatis and N. gonorrhoeae in women, in diverse settings and populations, in sub-Saharan Africa from 2005 to 2014. These studies were all conducted in countries with a moderate to high HIV prevalence and show that these bacterial STIs are common among women.
We observed a considerable difference in the number of studies conducted in each sub-Saharan country with the majority of studies conducted in South Africa (18\%), Tanzania (17\%) and Kenya $(12 \%)$. Furthermore, the publications that we identified only represented approximately half of all sub-Saharan African countries; there were no data points for a considerable number of countries. The limited attention paid to the epidemiology of chlamydial infection and gonorrhoea in sub-Saharan African countries is further reflected by a total of only seven studies reporting on incidence estimates of $C$. trachomatis or $N$. gonorrhoeae infections between 2005 and 2014. However, although we included several databases in this analysis, we cannot rule out existence of (unpublished) reports on STI prevalence or incidence.

The weighted prevalence of chlamydial infection and gonorrhoea for women recruited in their community $(3.9 \%$ and $2.2 \%)$ is in line with the WHO 2005 estimates $(3.9 \%$ and $2.1 \%$ ) published in 2008 based on modelling, although prevalence varied noticeably among sub-Saharan African countries. The subsequent WHO 2008 estimates published in 2012 for C. trachomatis infection are lower $(2.6 \%)$ than observed in our study, but in a similar range for $N$. gonorrhoeae $(2.3 \%) .{ }^{1,138}$ The most recent WHO estimates published in 2015 are similar for chlamydia $(3.7 \%)$, but lower for gonorrhoea $(1.7 \%){ }^{139}$ However, we observed an higher weighted prevalence among women recruited at PHC settings in sub-Saharan Africa $(6.3 \%$ and $4.3 \%)$ as compared to $\mathrm{WHO}$ estimates and results from community-based studies. There could be several reasons for this observed difference. First, publication bias might have occurred as studies that observe relatively high prevalence are more likely to be published, contributing to a higher prevalence in our analysis. Second, the WHO uses case-reporting surveillance systems in many countries which might underestimate the burden of asymptomatic infections. Although the WHO estimates are adjusted for unreported and undiagnosed cases, the question remains to what 
extent it should be adjusted, especially in case of $C$. trachomatis infections. Third, women with symptomatic STIs may be more likely to visit healthcare facilities for care than those without symptoms. Finally, our analyses included studies until 2014 whereas the estimates of the WHO are calculated over an earlier reference period.

Surprisingly, we observed a lower prevalence $(5.5 \%)$ of $C$. trachomatis infection among FSWs and a higher prevalence of $N$. gonorrhoeae $(7.6 \%)$ as compared to women recruited at $\mathrm{PHC}$ settings, but similar to women in community-based studies. Successful prevention programmes aimed at this specific population have been reported in some countries, including Ghana and Benin, resulting in decreased prevalence of these bacterial STIs. ${ }^{109,140}$ On the other hand, selection bias may have played a role in these studies. Sex workers constitute a key population and it may not be easy to recruit self-identified sex workers into a research study. It is not unthinkable that sex workers engaging in the highest risk behaviour for contracting STIs were not included in these studies.

The prevalence of $C$. trachomatis was higher, but associated with increased $N$. gonorrhoeae prevalence in all studies. The WHO prevalence estimates also suggest higher $C$. trachomatis infection in both 2005 and 2008, although chlamydial infection is suggested to show a decreasing trend over time while gonorrhoea is increasing. The strong associations observed in our analysis could contribute to better surveillance as it could facilitate prediction of burden of both STIs in settings where only one of these infections can be measured.

$N$. gonorrhoeae prevalence was associated with a higher HIV prevalence among women overall in studies included in our analysis, but for C. trachomatis we did not observe this relationship. Furthermore, at countrylevel, neither infection was associated with HIV prevalence as reported by UNAIDS (for adults) among women recruited at the diverse settings. Although it is biologically plausible that both infections enhance HIV acquisition and transmission there are several reasons that could explain the observed lack of association. First, the data retrieved from studies are fairly heterogeneous as study population (and size), country and location varied, which could have weakened the relation. Second, we used most recent UNAIDS HIV estimates while study year of many studies was prior to this date. Third, successful HIV prevention programmes have succeeded in decreasing the prevalence of $C$. trachomatis and $N$. gonorrhoeae. ${ }^{109,140}$ A last important reason could be the potential difference in timeline as one could have become infected with HIV years ago and have recently become infected or reinfected with $C$. trachomatis or $N$. gonorrhoeae.
Prevalence of chlamydial infection and gonorrhoea at community level was higher in sub-Saharan African countries with relatively higher reported HDI (by UNDP). We did not observe the same association for studies of women recruited in PHC settings. Despite the fact that poverty is associated with increased vulnerability for STIs in general, the observation may also suggest that sexual risk behaviour may be higher in areas in Africa of relatively higher socio-economic status, for example through increased alcohol use. Another explanation could be that these results are reflecting that countries with higher HDI (e.g. South Africa) have better resources to educate women at risk for STIs at the community level and mobilize women with symptoms or as part of partner notification to be tested. On the other hand, exposure to specific public health programmes may possibly have affected this association, for example mass treatment of trachoma in some of the countries ranking relatively low on HDI.

There are very limited data on oral and anal infections in African women; only one study observed considerable anorectal prevalence of $C$. trachomatis and $N$. gonorrhoeae among women visiting a PHC clinic. Recent studies from Europe show treatment of anal $C$. trachomatis and $N$. gonorrhoeae in women will likely reduce transmission of these STIs. ${ }^{141,142}$ As there are limited data available, more research is warranted to determine the role and burden of these infections in sub-Saharan Africa.

In line with literature we observed higher prevalence of $N$. gonorrhoeae among women recruited at PHC settings by using NAAT as the detection method compared to culture. This may be explained by logistic and operational challenges in culturing $N$. gonorrhoeae, the bacterial frailty and the known higher sensitivity of molecular assays. ${ }^{143-145}$ This observation has implications for STI control programmes in these settings. Given lower performance of culture compared to molecular techniques, studies and health policies using culture are likely to underestimate the burden of STIs.

This study has several limitations. First, there was considerable heterogeneity of publications included in this analysis. Study design, sample size, sampling strategy, laboratory method and specimen type used varied substantially across the included studies. Each of these studies has its own limitations and risk of bias; the sampling strategy (e.g. convenience sampling) used in some studies may have accounted for some of the outliers. By using clear inclusion criteria and calculating prevalence estimates per population group we tried to limit this limitation. However, heterogeneity of studies should be taken into account when interpreting our results. A second limitation was the availability of studies per country within sub-Saharan Africa as only half 
of the countries had one or more studies available. Local variations in sexual behaviour might result in differences between countries in sub-Saharan Africa that could not be identified due to a limited number of countries with sufficient data. Third, we did not include conference abstracts and grey literature in this evaluation which may have affected the breadth of our search. Also, our search may have missed a few studies with STI data embedded as part of a different objective, e.g. HIV clinical trial, neither can we rule out the existence of unpublished country-level data. A further possible limitation is that settings where chlamydial infection and gonorrhoea are regarded as public health concerns, and thus more effectively integrated in healthcare provision, are potentially more likely to be targeted by epidemiological studies. Likewise, more studies were conducted in urban than in rural settings. Finally, publication bias may have occurred in favour of high-prevalence studies resulting in an overestimation of weighted prevalence in our analysis

The WHO states that an important obstacle to provision of services for control of STIs at the decisionmaking level is that policy-makers give low priority to control of STIs, which is potentially aggravated by the stigmatization and prejudice associated with these infections and limited awareness of the importance of their impact on health and economic development. ${ }^{146}$ Although STIs other than HIV are not named in either the Millennium Development Goals or the new Sustainable Development Goals, they have important global health priorities on their own, but their impact is unrecognized. ${ }^{147}$

The Disease Control Priorities Project (DCPP) considered the diagnosis and treatment of STIs as a neglected low-cost opportunity to improve health in sub-Saharan Africa. ${ }^{148}$ However, many countries fail to provide suitable education and services to populations at risk for STIs. ${ }^{146}$ In order to overcome these obstacles enhanced surveillance of STIs is required in sub-Saharan Africa in order to stronger advocate the importance of STI control and facilitate the integration of STI control measures in existing reproductive health programmes.

\section{Brief conclusions}

- C. trachomatis and N. gonorrhoeae constitute a major public health problem among women, but the burden of infection in sub-Saharan Africa is poorly documented.

- Our overview underlines a relevant prevalence of genital $C$. trachomatis and $N$. gonorrhoeae in women in diverse settings in sub-Saharan Africa with geographic differences.
- Only a few studies reported on oral and anal infections.

- Enhanced surveillance of STIs is required and better control strategies are warranted to reduce the burden of infection and prevent long-term complications of these infections.

\section{Declaration of conflicting interests}

The author(s) declared no potential conflicts of interest with respect to the research, authorship, and/or publication of this article.

\section{Funding}

The author(s) received no financial support for the research, authorship, and/or publication of this article.

\section{References}

1. World Health Organization. Global incidence and prevalence of selected curable sexually transmitted infections - 2008. World Health Organization library, http://apps. who.int/iris/bitstream/10665/75181/1/9789241503839_ eng.pdf (2012, accessed 1 February 2016).

2. Kenyon C, Buyze $\mathbf{J}$ and Colebunders R. Classification of incidence and prevalence of certain sexually transmitted infections by world regions. Int J Infect Dis 2014; 18: 73-80.

3. Comparison of female to male and male to female transmission of HIV in 563 stable couples. European Study Group on Heterosexual Transmission of HIV. BMJ 1992; 304: 809-813.

4. Lin JS, Donegan SP, Heeren TC, et al. Transmission of Chlamydia trachomatis and Neisseria gonorrhoeae among men with urethritis and their female sex partners. J Infect Dis 1998; 178: 1707-1712.

5. Blanc AK. The effect of power in sexual relationships on sexual and reproductive health: an examination of the evidence. Stud Fam Plann 2001; 32: 189-213.

6. Peters RP, Dubbink JH, van der Eem L, et al. Crosssectional study of genital, rectal, and pharyngeal chlamydia and gonorrhea in women in rural South Africa. Sex Transm Dis 2014; 41: 564-569.

7. Romoren M, Sundby J, Velauthapillai M, et al. Chlamydia and gonorrhoea in pregnant Batswana women: time to discard the syndromic approach? BMC Infect Dis 2007; 7: 27.

8. Gray-Swain MR and Peipert JF. Pelvic inflammatory disease in adolescents. Curr Opin Obstet Gynecol 2006; 18: $503-510$.

9. Fleming DT and Wasserheit JN. From epidemiological synergy to public health policy and practice: the contribution of other sexually transmitted diseases to sexual transmission of HIV infection. Sex Transm Infect 1999; 75: 3-17.

10. Ding J, Rapista A, Teleshova N, et al. Neisseria gonorrhoeae enhances HIV-1 infection of primary resting CD4+ $\mathrm{T}$ cells through TLR2 activation. J Immunol 2010; 184: 2814-2824. 
11. Mlisana K, Naicker N, Werner L, et al. Symptomatic vaginal discharge is a poor predictor of sexually transmitted infections and genital tract inflammation in highrisk women in South Africa. J Infect Dis 2012; 206: 6-14.

12. Trollope-Kumar K and Guyatt G. Syndromic approach for treatment of STIs: time for a change. Lancet 2006; 367: 1380-1381.

13. Pham-Kanter GB, Steinberg $\mathrm{MH}$ and Ballard RC. Sexually transmitted diseases in South Africa. Genitourin Med 1996; 72: 160-171.

14. Johnson LF, Coetzee DJ and Dorrington RE. Sentinel surveillance of sexually transmitted infections in South Africa: a review. Sex Transm Infect 2005; 81: 287-293.

15. Cwikel JG, Lazer T, Press F, et al. Sexually transmissible infections among female sex workers: an international review with an emphasis on hard-to-access populations. Sex Heal 2008; 5: 9-16.

16. Liberati A, Altman DG, Tetzlaff J, et al. The PRISMA statement for reporting systematic reviews and metaanalyses of studies that evaluate healthcare interventions: explanation and elaboration. BMJ 2009; 339: b2700.

17. World Health Organization. WHO country offices in the WHO African region. World Health Organization library, www.afro.who.int/en/countries.html (2013, accessed 15 January 2016).

18. The World Bank Group. Sub-Saharan Africa, http:// data.worldbank.org/region/SSA (2014, accessed 15 January 2016).

19. Darj E, Mirembe FM and Rassjo EB. STI-prevalence and differences in social background and sexual behavior among urban and rural young women in Uganda. Sex Reprod Healthc 2010; 1: 111-115.

20. de Walque WD, Dow WH, Nathan R, et al. Incentivising safe sex: a randomised trial of conditional cash transfers for HIV and sexually transmitted infection prevention in rural Tanzania. BMJ Open 2012; 2: e000747.

21. United Nations Development Programme. Human Development Index (HDI), http://hdr.undp.org/en/con tent/human-development-index-hdi (2015, accessed 1 February 2016).

22. UNAIDS. People living with HIV; HIV prevalence among adults (15-49), http://aidsinfo.unaids.org (2014, accessed 15 January 2016).

23. UNAIDS. Sex workers; HIV prevalence in sex workers, http://aidsinfo.unaids.org (2014, accessed 15 January 2016).

24. Ramjee G and Wand H. Geographical clustering of high risk sexual behaviors in 'hot-spots' for HIV and sexually transmitted infections in Kwazulu-Natal, South Africa. AIDS Behav 2014; 18: 317-322.

25. Naidoo S, Wand H, Abbai NS, et al. High prevalence and incidence of sexually transmitted infections among women living in Kwazulu-Natal, South Africa. AIDS Res Ther 2014; 11: 31.

26. Behets FM, Van DK, Rasamindrakotroka A, et al. Socio-demographic and behavioural factors associated with high incidence of sexually transmitted infections in female sex workers in Madagascar following presumptive therapy. Sex Heal 2005; 2: 77-84.

27. Braunstein SL, Ingabire CM, Kestelyn E, et al. High human immunodeficiency virus incidence in a cohort of Rwandan female sex workers. Sex Transm Dis 2011; 38: 385-394.

28. Cohen CR, Nosek M, Meier A, et al. Mycoplasma genitalium infection and persistence in a cohort of female sex workers in Nairobi, Kenya. Sex Transm Dis 2007; 34: 274-279.

29. Cowan FF, Pascoe SJ, Barlow KL, et al. Association of genital shedding of herpes simplex virus type 2 and HIV-1 among sex workers in rural Zimbabwe. AIDS 2006; 20: 261-267.

30. Detels R, Green AM, Klausner JD, et al. The incidence and correlates of symptomatic and asymptomatic Chlamydia trachomatis and Neisseria gonorrhoeae infections in selected populations in five countries. Sex Transm Dis 2011; 38: 503-509.

31. Hoke TH, Feldblum PJ, Van DK, et al. Temporal trends in sexually transmitted infection prevalence and condom use following introduction of the female condom to Madagascar sex workers. Int $J$ STD AIDS 2007; 18: 461-466.

32. Kapina M, Reid C, Roman K, et al. HIV incidence rates and risk factors for urban women in Zambia: preparing for a microbicide clinical trial. Sex Transm Dis 2009; 36: 129-133.

33. Kehinde AO and Lawoyin TO. Prevalence of STI/HIV co-infections among special treatment clinic attendees in Ibadan, Nigeria. $J$ R Soc Promot Health 2005; 125: 186-190.

34. Lemme F, Doyle AM, Changalucha J, et al. HIV infection among young people in northwest Tanzania: the role of biological, behavioural and socio-demographic risk factors. PLoS One 2013; 8: e66287.

35. Mavedzenge SN, Weiss HA, Montgomery ET, et al. Determinants of differential HIV incidence among women in three southern African locations. J Acquir Immune Defic Syndr 2011; 58: 89-99.

36. Minnis AM, van der Straten A, Gerdts C, et al. A comparison of four condom-use measures in predicting pregnancy, cervical STI and HIV incidence among Zimbabwean women. Sex Transm Infect 2010; 86: 231-235.

37. Olsen B, Mansson F, Camara C, et al. Phenotypic and genetic characterisation of bacterial sexually transmitted infections in Bissau, Guinea-Bissau, West Africa: a prospective cohort study. BMJ Open 2012; 2: e000636.

38. Pettifor A, Delany S, Kleinschmidt I, et al. Use of injectable progestin contraception and risk of STI among South African women. Contraception 2009; 80: 555-560.

39. Pettifor AE, Turner AN, Van DK, et al. Increased risk of chlamydial and gonococcal infection in adolescent sex workers in Madagascar. Sex Transm Dis 2007; 34: 475-478. 
40. Rassjö EB, Mirembe FM and Darj E. Vulnerability and risk factors for sexually transmitted infections and HIV among adolescents in Kampala, Uganda. AIDS Care 2006; 18: 710-716.

41. Rassjo EB, Mirembe F and Darj E. Self-reported sexual behaviour among adolescent girls in Uganda: reliability of data debated. Afr Health Sci 2011; 11: 383-389.

42. Richardson BA, Kelly C, Ramjee G, et al. Appropriateness of hydroxyethylcellulose gel as a placebo control in vaginal microbicide trials: a comparison of the two control arms of HPTN. J Acquir Immune Defic Syndr 2013; 63: 120-125.

43. Turner AN, Feldblum $P$ and Hoke TH. Condom use and sexually transmitted infections among Malagasy sex workers. Int J STD AIDS 2011; 22: 552-557.

44. Vandepitte J, Muller E, Bukenya J, et al. Prevalence and correlates of Mycoplasma genitalium infection among female sex workers in Kampala, Uganda. J Infect Dis 2012; 205: 289-296.

45. Vandepitte J, Weiss HA, Bukenya J, et al. Alcohol use, mycoplasma genitalium, and other STIs associated With HIV incidence among women at high risk in Kampala, Uganda. J Acquir Immune Defic Syndr 2013; 62: 119-126.

46. Vandepitte J, Weiss HA, Kyakuwa N, et al. Natural history of Mycoplasma genitalium infection in a cohort of female sex workers in Kampala, Uganda. Sex Transm Dis 2013; 40: 422-427.

47. Veldhuijzen NJ, Braunstein SL, Vyankandondera J, et al. The epidemiology of human papillomavirus infection in HIV-positive and HIV-negative high-risk women in Kigali, Rwanda. BMC Infect Dis 2011; 11: 333.

48. Venkatesh KK, van der Straten A, Mayer KH, et al. African women recently infected with HIV-1 and HSV-2 have increased risk of acquiring Neisseria gonorrhoeae and Chlamydia trachomatis in the methods for improving reproductive health in Africa trial. Sex Transm Dis 2011; 38: 562-570.

49. Venkatesh KK, van der Straten A, Cheng H, et al. The relative contribution of viral and bacterial sexually transmitted infections on HIV acquisition in southern African women in the methods for improving reproductive health in Africa study. Int $J$ STD AIDS 2011; 22: 218-224.

50. Vandepitte J, Bukenya J, Hughes P, et al. Clinical characteristics associated with Mycoplasma genitalium infection among women at high risk of HIV and other STI in Uganda. Sex Transm Dis 2012; 39: 487-491.

51. Reddy P, Saleh-Onoya D, Sifunda S, et al. Preference for dry sex, condom use and risk of STI among HIVnegative black women in the Western Cape province, South Africa. S Afr J Sci 2009; 105: 2-73.

52. Celentano DD, Mayer KH, Pequegnat W, et al. Prevalence of sexually transmitted diseases and risk behaviors from the NIMH collaborative HIV/STD prevention trial. Int J Sex Health 2010; 22: 272-284.

53. Downs JA, van Dam GJ, Changalucha JM, et al. Association of Schistosomiasis and HIV infection in Tanzania. Am J Trop Med Hyg 2012; 87: 868-873.
54. Doyle AM, Ross DA, Maganja K, et al. Long-term biological and behavioural impact of an adolescent sexual health intervention in Tanzania: follow-up survey of the community-based MEMA kwa Vijana Trial. PLoS Med 2010; 7: e1000287.

55. Franceschi S, Smith JS, van den Brule A, et al. Cervical infection with Chlamydia trachomatis and Neisseria gonorrhoeae in women from ten areas in four continents. A cross-sectional study. Sex Transm Dis 2007; 34: 563-569.

56. Ghebremichael M, Paintsil E and Larsen U. Alcohol abuse, sexual risk behaviors, and sexually transmitted infections in women in Moshi urban district, northern Tanzania. Sex Transm Dis 2009; 36: 102-107.

57. Gueye NA, Faye CM, Ndiaye I, et al. [Screening for HIV, syphilis, Chlamydia trachomatis and Neisseria gonorrhoeae during a combined survey conducted in Malicouna, a Senegalese rural area]. Bull Soc Pathol Exot 2009; 102: 150-154.

58. Guffey MB, Richardson B, Husnik M, et al. HPTN 035 phase II/IIb randomised safety and effectiveness study of the vaginal microbicides BufferGel and $0.5 \%$ PRO 2000 for the prevention of sexually transmitted infections in women. Sex Transm Infect 2014; 90: 363-369.

59. Halpern V, Ogunsola F, Obunge O, et al. Effectiveness of cellulose sulfate vaginal gel for the prevention of HIV infection: results of a Phase III trial in Nigeria. PLoS One 2008; 3: e3784.

60. Hazel A, Ponnaluri-Wears S, Davis GS, et al. High prevalence of Neisseria gonorrhoeae in a remote, undertreated population of Namibian pastoralists. Epidemiol Infect 2014; 142: 2422-2432.

61. Kapiga S, Kelly C, Weiss S, et al. Risk factors for incidence of sexually transmitted infections among women in South Africa, Tanzania, and Zambia: results from HPTN 055 study. Sex Transm Dis 2009; 36: 199-206.

62. Leutscher P, Jensen JS, Hoffmann S, et al. Sexually transmitted infections in rural Madagascar at an early stage of the HIV epidemic: a 6-month community-based follow-up study. Sex Transm Dis 2005; 32: 150-155.

63. Mensch BS, Hewett PC, Gregory R, et al. Sexual behavior and STI/HIV status among adolescents in rural Malawi: an evaluation of the effect of interview mode on reporting. Stud Fam Plann 2008; 39: 321-334.

64. Ogilvie GS, Mitchell S, Sekikubo M, et al. Results of a community-based cervical cancer screening pilot project using human papillomavirus self-sampling in Kampala, Uganda. Int J Gynaecol Obstet 2013; 122: 118-123.

65. Otieno FO, Ndivo R, Oswago S, et al. Correlates of prevalent sexually transmitted infections among participants screened for an HIV incidence cohort study in Kisumu, Kenya. Int J STD AIDS 2015; 26: 225-237.

66. Paz-Soldan VA, Hoffman I, deGraft J, et al. Sexually transmitted infection (STI) screening, case and contact treatment, and condom promotion resulting in STI reduction two years later in rural Malawi. Malawi Med J 2012; 24: 8-13.

67. Rutherford GW, Anglemyer A, Bagenda D, et al. University students and the risk of HIV and other 
sexually transmitted infections in Uganda: the Crane survey. Int J Adolesc Med Health 2014; 26: 209-215.

68. Vallely A, Kasindi S, Hambleton IR, et al. Microbicides development program, Tanzania-baseline characteristics of an occupational cohort and reattendance at 3 months. Sex Transm Dis 2007; 34: 638-643.

69. Yirenya-Tawiah D, Annang TN, Apea-Kubi KA, et al. Chlamydia trachomatis and Neisseria gonorrhoeae prevalence among women of reproductive age living in urogenital schistosomiasis endemic area in Ghana. BMC Res Notes 2014; 7: 349.

70. Aboud S, Msamanga G, Read JS, et al. Genital tract infections among HIV-infected pregnant women in Malawi, Tanzania and Zambia. Int J STD AIDS 2008; 19: 824-832.

71. Alcaide ML, Jones DL, Chitalu N, et al. Chlamydia and gonorrhea infections in HIV-positive women in Urban Lusaka, Zambia. J Glob Infect Dis 2012; 4: 141-144.

72. Bukusi EA, Cohen CR, Meier AS, et al. Bacterial vaginosis: risk factors among Kenyan women and their male partners. Sex Transm Dis 2006; 33: 361-367.

73. Chalamilla G, Mbwana J, Mhalu F, et al. Patterns of sexually transmitted infections in adolescents and youth in Dar es Salaam, Tanzania. BMC Infect Dis 2006; 6: 22.

74. Chiduo M, Theilgaard ZP, Bakari V, et al. Prevalence of sexually transmitted infections among women attending antenatal clinics in Tanga, north eastern Tanzania. Int $J$ STD AIDS 2012; 23: 325-329.

75. Frohlich JA, Abdool KQ, Mashego MM, et al. Opportunities for treating sexually transmitted infections and reducing HIV risk in rural South Africa. $J$ Adv Nurs 2007; 60: 377-383.

76. Govender S, Theron GB, Odendaal HJ, et al. Prevalence of genital mycoplasmas, ureaplasmas and chlamydia in pregnancy. J Obstet Gynaecol 2009; 29: 698-701.

77. Guimaräes H, Castro R, Tavora TL, et al. Assessing therapeutic management of vaginal and urethral symptoms in an anonymous HIV testing centre in Luanda, Angola. J Infect Dev Ctries 2013; 7: 720-725.

78. Guthrie BL, Kiarie JN, Morrison S, et al. Sexually transmitted infections among HIV-1-discordant couples. PLoS One 2009; 4: e8276.

79. Hubacher D, Raymond ER, Beksinska M, et al. Hormonal contraception and the risks of STI acquisition: results of a feasibility study to plan a future randomized trial. Contraception 2008; 77: 366-370.

80. Jones HE, Altini L, de KA, et al. Home-based versus clinic-based self-sampling and testing for sexually transmitted infections in Gugulethu, South Africa: randomised controlled trial. Sex Transm Infect 2007; 83: 552-557.

81. Kinoshita-Moleka R, Smith JS, Atibu J, et al. Low prevalence of HIV and other selected sexually transmitted infections in 2004 in pregnant women from Kinshasa, the Democratic Republic of the Congo. Epidemiol Infect 2008; 136: 1290-1296.

82. Kleinschmidt I, Rees H, Delany S, et al. Injectable progestin contraceptive use and risk of HIV infection in a South African family planning cohort. Contraception 2007; 75: 461-467.
83. Lassey AT, Newman MJ and Opintan JA. Vaginal flora of first time urban family planning attendants in Accra, Ghana. West Afr J Med 2005; 24: 219-222.

84. Lewis DA, Chirwa TF, Msimang VM, et al. Urethritis/ cervicitis pathogen prevalence and associated risk factors among asymptomatic HIV-infected patients in South Africa. Sex Transm Dis 2012; 39: 531-536.

85. Luján J, de Onate WA, Delva W, et al. Prevalence of sexually transmitted infections in women attending antenatal care in Tete province, Mozambique. $S$ Afr Med $J$ 2008; 98: 49-51.

86. Mansson F, Camara C, Biai A, et al. High prevalence of HIV-1, HIV-2 and other sexually transmitted infections among women attending two sexual health clinics in Bissau, Guinea-Bissau, West Africa. Int J STD AIDS 2010; 21: 631-635.

87. Marx G, John-Stewart G, Bosire R, et al. Diagnosis of sexually transmitted infections and bacterial vaginosis among HIV-1-infected pregnant women in Nairobi. Int J STD AIDS 2010; 21: 549-552.

88. Melo J, Folgosa E, Manjate D, et al. Low prevalence of HIV and other sexually transmitted infections in young women attending a youth counselling service in Maputo, Mozambique. Trop Med Int Health 2008; 13: 17-20.

89. Mhlongo S, Magooa P, Muller EE, et al. Etiology and STI/HIV coinfections among patients with urethral and vaginal discharge syndromes in South Africa. Sex Transm Dis 2010; 37: 566-570.

90. Msuya SE, Mbizvo EM, Stray-Pedersen B, et al. Risk assessment at the primary health care level in Moshi, Tanzania: limits in predicting sexually transmitted infections among women. Cent Afr J Med 2006; 52: 97-104.

91. Msuya SE, Uriyo J, Hussain A, et al. Prevalence of sexually transmitted infections among pregnant women with known HIV status in northern Tanzania. Reprod Health 2009; 6: 4.

92. Nwadioha S, Egah D, Nwokedi E, et al. A study of female genital swabs in primary health care centres in Jos, Nigeria. Asian Pac J Trop Dis 2011; 1: 52-54.

93. Onoya D, Reddy P, Sifunda S, et al. Transactional sexual relationships, sexually transmitted infection risk, and condom use among young Black Women in peri-urban areas of the Western Cape Province of South Africa. Womens Health Issues 2012; 22: e277-e282.

94. Paz-Bailey G, Rahman M, Chen C, et al. Changes in the etiology of sexually transmitted diseases in Botswana between 1993 and 2002: implications for the clinical management of genital ulcer disease. Clin Infect Dis 2005; 41: 1304.

95. Pettifor AE, Kleinschmidt I, Levin J, et al. A community-based study to examine the effect of a youth HIV prevention intervention on young people aged 15-24 in South Africa: results of the baseline survey. Trop Med Int Health 2005; 10: 971-980.

96. Pickering JM, Whitworth JA, Hughes P, et al. Aetiology of sexually transmitted infections and response to syndromic treatment in southwest Uganda. Sex Transm Infect 2005; 81: 488-493. 
97. Ramjee G, van der Straten A, Chipato T, et al. The diaphragm and lubricant gel for prevention of cervical sexually transmitted infections: results of a randomized controlled trial. PLoS One 2008; 3: e3488.

98. Rassjö EB, Kambugu F, Tumwesigye MN, et al. Prevalence of sexually transmitted infections among adolescents in Kampala, Uganda, and theoretical models for improving syndromic management. $J$ Adolesc Health 2006; 38: 213-221.

99. Saleh-Onoya D, Reddy PS, Ruiter RA, et al. Condom use promotion among isiXhosa speaking women living with HIV in the Western Cape Province, South Africa: a pilot study. AIDS Care 2009; 21: 817-825.

100. Singa B, Glick SN, Bock N, et al. Sexually transmitted infections among HIV-infected adults in HIV care programs in Kenya: a national sample of HIV clinics. Sex Transm Dis 2013; 40: 148-153.

101. Tann CJ, Mpairwe H, Morison L, et al. Lack of effectiveness of syndromic management in targeting vaginal infections in pregnancy in Entebbe, Uganda. Sex Transm Infect 2006; 82: 285-289.

102. van de Wijgert J, Altini L, Jones H, et al. Two methods of self-sampling compared to clinician sampling to detect reproductive tract infections in Gugulethu, South Africa. Sex Transm Dis 2006; 33: 516-523.

103. van de Wijgert JH, Morrison CS, Brown J, et al. Disentangling contributions of reproductive tract infections to HIV acquisition in African Women. Sex Transm Dis 2009; 36: 357-364.

104. Watson-Jones D, Weiss HA, Changalucha JM, et al. Adverse birth outcomes in United Republic of Tanzania-impact and prevention of maternal risk factors. Bull World Health Organ 2007; 85: 9-18.

105. Aho J, Koushik A, Coutlee F, et al. Prevalence of HIV, human papillomavirus type 16 and herpes simplex virus type 2 among female sex workers in Guinea and associated factors. Int J STD AIDS 2014; 25: 280-288.

106. Ahoyo AB, Alary M, Meda H, et al. [Female sex workers in Benin 2002. Behavioural survey and HIV and other STI screening]. Sante 2007; 17: 143-151.

107. Ahoyo AB, Alary M, Ndour M, et al. [HIV and sexually transmitted disease among female sex workers in Benin]. Med Trop (Mars) 2009; 69: 457-462.

108. Alary M, Gbenafa-Agossa C, Aina G, et al. Evaluation of a rapid point-of-care test for the detection of gonococcal infection among female sex workers in Benin. Sex Transm Infect 2006; 82: v29-v32.

109. Béhanzin L, Diabate S, Minani I, et al. Decline in the prevalence of HIV and sexually transmitted infections among female sex workers in Benin over 15 years of targeted interventions. J Acquir Immune Defic Syndr 2013; 63: 126-134.

110. Chersich MF, Luchters SM, Malonza IM, et al. Heavy episodic drinking among Kenyan female sex workers is associated with unsafe sex, sexual violence and sexually transmitted infections. Int J STD AIDS 2007; 18: 764-769.

111. Cohen CR, Koochesfahani KM, Meier AS, et al. Immunoepidemiologic profile of Chlamydia trachomatis infection: importance of heat-shock protein 60 and interferon-gamma. J Infect Dis 2005; 192: 591-599.

112. Cowan FM, Hargrove JW, Langhaug LF, et al. The appropriateness of core group interventions using presumptive periodic treatment among rural Zimbabwean women who exchange sex for gifts or money. $J$ Acquir Immune Defic Syndr 2005; 38: 202-207.

113. Dunkle KL, Beksinska ME, Rees VH, et al. Risk factors for HIV infection among sex workers in Johannesburg, South Africa. Int J STD AIDS 2005; 16: 256-261.

114. Feldblum PJ, Hatzell T, Van DK, et al. Results of a randomised trial of male condom promotion among Madagascar sex workers. Sex Transm Infect 2005; 81: 166-173.

115. Gomih-Alakija A, Ting J, Mugo N, et al. Clinical characteristics associated with Mycoplasma genitalium among female sex workers in Nairobi, Kenya. J Clin Microbiol 2014; 52: 3660-3666.

116. Harijaona V, Ramambason JD, Morisset R, et al. Prevalence of and risk factors for sexually-transmitted infections in hidden female sex workers. Med Mal Infect 2009; 39: 909-913.

117. Izulla P, McKinnon LR, Munyao J, et al. HIV postexposure prophylaxis in an urban population of female sex workers in Nairobi, Kenya. J Acquir Immune Defic Syndr 2013; 62: 220-225.

118. Mamadou S, Laouel KA, Rabiou S, et al. [Prevalence of the HIV infection and five other sexually-transmitted infections among sex workers in Niamey, Niger]. Bull Soc Pathol Exot 2006; 99: 19-22.

119. Masese L, Baeten JM, Richardson BA, et al. Incidence and correlates of Chlamydia trachomatis infection in a high-risk cohort of Kenyan women. Sex Transm Dis 2013; 40: 221-225.

120. Pépin J, Labbe AC, Khonde N, et al. Mycoplasma genitalium: an organism commonly associated with cervicitis among west African sex workers. Sex Transm Infect 2005; 81: 67-72.

121. Vandepitte J, Bukenya J, Weiss HA, et al. HIV and other sexually transmitted infections in a cohort of women involved in high-risk sexual behavior in Kampala, Uganda. Sex Transm Dis 2011; 38: 316-323.

122. Vandepitte JM, Malele F, Kivuvu DM, et al. HIV and other sexually transmitted infections among female sex workers in Kinshasa, Democratic Republic of Congo, in 2002. Sex Transm Dis 2007; 34: 203-208.

123. Vuylsteke B, Semde G, Sika L, et al. HIV and STI prevalence among female sex workers in Cote d'Ivoire: why targeted prevention programs should be continued and strengthened. PLoS One 2012; 7: e32627.

124. Veldhuijzen NJ, van SM, Nyinawabega $J$, et al. Prevalence of sexually transmitted infections, genital symptoms and health-care seeking behaviour among HIV-negative female sex workers in Kigali, Rwanda. Int J STD AIDS 2013; 24: 139-143.

125. Buhalata SN, Kwesigabo G, Sembuche S, et al. Genital tract infections in women attending sexually transmitted infection clinics in Mwanza, north-west Tanzania. South Afr J Epidemiol Infect 2013; 28: 48-54. 
126. Demba E, Morison L, van der Loeff MS, et al. Bacterial vaginosis, vaginal flora patterns and vaginal hygiene practices in patients presenting with vaginal discharge syndrome in The Gambia, West Africa. BMC Infect Dis 2005; 5: 12.

127. Kehinde AO and Lawoyin TO. STI/HIV co-infections in UCH, Ibadan, Nigeria. Afr J Reprod Health 2005; 9: 42-48.

128. Manhart LE, Mostad SB, Baeten JM, et al. High Mycoplasma genitalium organism burden is associated with shedding of HIV-1 DNA from the cervix. I Infect Dis 2008; 1;197: 733-736.

129. Masson L, Mlisana K, Little F, et al. Defining genital tract cytokine signatures of sexually transmitted infections and bacterial vaginosis in women at high risk of HIV infection: a cross-sectional study. Sex Transm Infect 2014; 90: 580-587.

130. Zimba TF, Apalata T, Sturm WA, et al. Aetiology of sexually transmitted infections in Maputo, Mozambique. J Infect Dev Ctries 2011; 5: 41-47.

131. Baisley K, Changalucha J, Weiss HA, et al. Bacterial vaginosis in female facility workers in north-western Tanzania: prevalence and risk factors. Sex Transm Infect 2009; 85: 370-375.

132. Francis SC, Ao TT, Vanobberghen FM, et al. Epidemiology of curable sexually transmitted infections among women at increased risk for HIV in northwestern Tanzania: inadequacy of syndromic management. PLoS One 2014; 9: e101221.

133. Kapiga SH, Sam NE, Masenga EJ, et al. Risk factors for bacterial vaginosis among bar and hotel workers in Northern Tanzania. East Afr Med J 2005; 82: 85-91.

134. Opoku BK and Sarkodie Y. Prevalence of genital chlamydia and gonococcal infections in at risk women in the kumasi metropolis, ghana. Ghana Med $J$ 2010; 44: $21-24$.

135. Riedner G, Hoffmann O, Rusizoka M, et al. Decline in sexually transmitted infection prevalence and HIV incidence in female barworkers attending prevention and care services in Mbeya Region, Tanzania. AIDS 2006; 20: 609 .

136. Tanton C, Weiss HA, Le GJ, et al. Correlates of HIV-1 genital shedding in Tanzanian women. PLoS One 2011; 6: e17480.

137. Low AJ, Konate I, Nagot N, et al. Neisseria gonorrhoeae and Chlamydia trachomatis infection in HIV-1infected women taking antiretroviral therapy: a prospective cohort study from Burkina Faso. Sex Transm Infect 2014; 90: 100-103.
138. World Health Organization. Prevalence and incidence of selected sexually transmitted infections. Chlamydia trachomatis, Neisseria gonorrhoeae, syphilis and Trichomonas vaginalis. Methods and results used by WHO to generate 2005 estimates, www.who.int/repro ductivehealth/publications/rtis/9789241502450/en/ (2014, accessed 1 August 2016).

139. Newman L, Rowley J, Vander HS, et al. Global estimates of the prevalence and incidence of four curable sexually transmitted infections in 2012 based on systematic review and global reporting. PLoS One 2015; 10: $\mathrm{e} 0143304$.

140. Wondergem P, Green K, Wambugu S, et al. A short history of HIV prevention programs for female sex workers in Ghana: lessons learned over 3 decades. J Acquir Immune Defic Syndr 2015; 68: S138-S145.

141. Dukers-Muijrers NH, Schachter J, van Liere GA, et al. What is needed to guide testing for anorectal and pharyngeal Chlamydia trachomatis and Neisseria gonorrhoeae in women and men? Evidence and opinion. BMC Infect Dis 2015; 15: 533.

142. Peters RP, Nijsten N, Mutsaers J, et al. Screening of oropharynx and anorectum increases prevalence of Chlamydia trachomatis and Neisseria gonorrhoeae infection in female STD clinic visitors. Sex Transm Dis 2011; 38: 783-787.

143. Land JA, Van Bergen JE, Morre SA, et al. Epidemiology of Chlamydia trachomatis infection in women and the cost-effectiveness of screening. Hum Reprod Update 2010; 16: 189-204.

144. Jespersen DJ, Flatten KS, Jones MF, et al. Prospective comparison of cell cultures and nucleic acid amplification tests for laboratory diagnosis of Chlamydia trachomatis infections. J Clin Microbiol 2005; 43: 5324-5326.

145. Recommendations for the laboratory-based detection of Chlamydia trachomatis and Neisseria gonorrhoeae-2014. MMWR Recomm Rep 2014; 63: 1-19.

146. World Health Organization. Global strategy for the prevention and control of sexually transmitted infections: 2006-2015. Breaking the chain of transmission, www. who.int/reproductivehealth/publications/rtis/ 9789241563475/en/ (2007, accessed 1 August 2016).

147. Glasier A, Gulmezoglu AM, Schmid GP, et al. Sexual and reproductive health: a matter of life and death. Lancet 2006; 368: 1595-1607.

148. Laxminarayan R, Mills AJ, Breman JG, et al. Advancement of global health: key messages from the Disease Control Priorities Project. Lancet 2006; 367 : 1193-1208. 